\title{
The Pareto Frontier of Inefficiency in Mechanism Design*
}

\author{
Aris Filos-Ratsikas ${ }^{\dagger} \quad$ Yiannis Giannakopoulos ${ }^{\ddagger} \quad$ Philip Lazos $^{\S}$
}

February 18, 2021

\begin{abstract}
We study the trade-off between the Price of Anarchy (PoA) and the Price of Stability (PoS) in mechanism design, in the prototypical problem of unrelated machine scheduling. We give bounds on the space of feasible mechanisms with respect to the above metrics, and observe that two fundamental mechanisms, namely the First-Price (FP) and the SecondPrice (SP), lie on the two opposite extrema of this boundary. Furthermore, for the natural class of anonymous task-independent mechanisms, we completely characterize the PoA/PoS Pareto frontier; we design a class of optimal mechanisms $\mathcal{S P} \mathcal{P}_{\alpha}$ that lie exactly on this frontier. In particular, these mechanisms range smoothly, with respect to parameter $\alpha \geq 1$ across the frontier, between the First-Price $\left(\mathcal{S P}_{1}\right)$ and Second-Price $\left(\mathcal{S P} \mathcal{P}_{\infty}\right)$ mechanisms.

En route to these results, we also provide a definitive answer to an important question related to the scheduling problem, namely whether non-truthful mechanisms can provide better makespan guarantees in the equilibrium, compared to truthful ones. We answer this question in the negative, by proving that the Price of Anarchy of all scheduling mechanisms is at least $n$, where $n$ is the number of machines.
\end{abstract}

\section{Introduction}

The field of algorithmic mechanism design was established in the seminal paper of Nisan and Ronen [44] and has ever since been at the centre of research in the intersection of economics and computer science. The research agenda put forward in [44] advocates the study of approximate solutions to interesting optimization problems, in settings where rational agents are in control of the input parameters. More concretely, the authors of [44] proposed a framework in which, not unlike classical approaches in approximation algorithms, algorithms that operate under certain limitations are evaluated in terms of their approximation ratio. In particular, in algorithmic mechanism design, this constraint comes from the requirement that agents should have the right incentives to always report their inputs truthfully. The corresponding algorithms, paired with appropriately chosen payment functions, are called mechanisms [43].

Another pioneering line of work, initiated by Koutsoupias and Papadimitriou [32] and popularized further by Roughgarden and Tardos [50], studies the inefficiency of games through the notion of the Price of Anarchy (PoA), which measures the deterioration of some objective at the worst-case Nash equilibrium. A more optimistic version of the same principle, where the

\footnotetext{
*Supported by ERC Advanced Grant 321171 (ALGAME), the Swiss National Science Foundation under contract No. 200021_165522 and the Alexander von Humboldt Foundation with funds from the German Federal Ministry of Education and Research (BMBF). Also partially supported by the ERC Advanced Grant 788893 AMDROMA "Algorithmic and Mechanism Design Research in Online Markets" and MIUR PRIN project ALGADIMAR "Algorithms, Games, and Digital Markets". Y. Giannakopoulos is an associated researcher with the Research Training Group GRK 2201 "Advanced Optimization in a Networked Economy", funded by the German Research Foundation (DFG).

An extended abstract of this paper appeared in WINE'19 [19].

${ }^{\dagger}$ University of Liverpool. Email: Aris.Filos-Ratsikas@liverpool.ac.uk

${ }^{\ddagger}$ TU Munich. Email: yiannis.giannakopoulos@tum.de

${ }^{\S}$ Sapienza University of Rome. Email: lazos@diag.uniroma1.it
} 
inefficiency is measured at the best equilibrium [53], was introduced in [1], under the name of Price of Stability (PoS).

Given the straightforward observation that mechanisms induce games between the agents that control their inputs, as well as the fact that truthfulness is typically a very demanding property, an alternative approach to the framework of Nisan and Ronen [44] is to design mechanisms that perform well in the equilibrium, i.e., they provide good PoA or PoS guarantees. This approach has been adopted, among others, by central papers in the field (e.g., see [51, 54] and references therein) and is by now as much a part of algorithmic mechanism design as the original framework of [44]. An interesting question that has arisen in many settings is whether non-truthful mechanisms (evaluated at the worst-case equilibrium, in terms of their PoA) can actually outperform truthful ones (evaluated at the truth-telling, dominant strategy equilibrium), for a given objective [11, 23, 31].

While the literature that studies the concepts of PoA and PoS is long and extensive, there seems to be a lack of a systematic approach investigating the trade-off between the two notions simultaneously. More concretely, given a problem in algorithmic mechanism design, it seems quite natural to explore not only the best mechanisms in terms of the two notions independently, but also the mechanisms that achieve the best trade-off between the two. In a sense, this approach concerns a "tighter" optimality notion, as among a set of mechanisms with an "acceptable" Price of Anarchy guarantee, we would like to identify the ones that provide the best possible Price of Stability. Our main contribution in the current paper is the proposal of such a research agenda and its application on the canonical problem in the field, introduced in the seminal work of Nisan and Ronen [44], that of scheduling on unrelated machines.

\subsection{Our Contributions}

PoA/PoS trade-off: We propose the research agenda of studying systematically the tradeoff between the Price of Anarchy and the Price of Stability in algorithmic mechanism design. Specifically, given a problem at hand and an objective function, we are interested in the trade-off between the PoA and the PoS of mechanisms for the given objective. We apply this approach on the prototypical problem of algorithmic mechanism design studied in [44], that of unrelated machine scheduling, where the machines are self-interested agents.

First, in Section 3, for the class of all possible mechanisms, we prove that PoA guarantees imply corresponding PoS lower bounds and vice-versa (Theorem 2), which allows us to quantify the possible trade-off between the two inefficiency notions in terms of a feasible region (see Fig. 2); we refer to the boundary of this region as the inefficiency boundary. Interestingly, two well-known mechanisms, namely the First-Price and the Second-Price mechanisms, turn out to lie on the extreme points of this boundary.

Next, in Section 4, for the well-studied class of task-independent and anonymous mechanisms ${ }^{1}$ we are able to show a tighter feasibility region (Theorem 6 ). As a matter of fact, its inefficiency boundary turns out to completely characterize the achievable trade-off between the PoA and the PoS: we design a class of mechanisms (Section 4.2) called $\mathcal{S P}_{\alpha}$, parameterized by a quantity $\alpha$, which are optimal in the sense that for any possible trade-off between the two inefficiency notions, there exists a mechanism in the class (i.e., an appropriate choice of $\alpha$ ) that exactly achieves this trade-off (Theorems 7 and 8). In other words, we obtain an exact description of the Pareto frontier of inefficiency (see Fig. 3).

Our $\mathcal{S P} \mathcal{P}_{\alpha}$ mechanisms are simple and intuitive and are based on the idea of setting reserve prices relatively to the declarations of the fastest machines. While this is clearly not truthful, we prove that it induces the equilibria which are desirable for our results. More precisely, the

\footnotetext{
${ }^{1}$ We remark that the best known mechanisms for several variants of truthful scheduling are task-independent and anonymous. In Section 5, we provide a more detailed discussion, as well as an almost matching trade-off bound for mechanisms that need not be anonymous (see Theorem 9).
} 
choice of $\alpha$ enables us to "control" the set of possible equilibria in a way that allows us to achieve any trade-off on the boundary.

The Price of Anarchy of scheduling: Our results also offer insights in an other interesting direction. The inefficiency boundary result for general mechanisms is based on a novel monotonicity lemma (Lemma 1), which is quite different from the well-known weak monotonicity property [52] (see, e.g., [9, 44]). Interestingly, we also use this lemma to prove a general lower bound of $n$ on the PoA of any mechanism for the scheduling problem (Theorem 1), where $n$ is the number of machines. This result contributes to the intriguing debate $[11,23,31]$ of whether general mechanisms (that may be non-truthful, evaluated at the worst-case equilibrium) can outperform truthful ones (evaluated at the truth-telling equilibrium). Given that the best known truthful mechanism achieves an $n$-approximation, our results here provide a definitive, negative answer to the aforementioned question (see Section 1.2.3 for a more detailed discussion). As a matter of fact, in Theorem 5 , we actually show that when evaluated at their worst-case equilibrium, truthful mechanisms are bound to perform even more poorly, as their PoA is unbounded.

Finally, in Section 5, we conclude with a detailed discussion, where we identify several intriguing directions for future work, both on a technical and a conceptual level.

\subsection{Related Work}

\subsubsection{The Algorithmic Scheduling Problem}

The algorithmic version of the scheduling problem (without any consideration to incentives) is one of the most fundamental problems in computer science, whose origins can be traced back to the works of Johnson [30], Jackson [29] and Graham [25]. The problem is often also generally referred to as the "Job Shop Scheduling Problem" [21], as it accurately models job assignment problems in manufacturing systems. On top of this connection, the machine scheduling problem in fact enjoys a plethora of applications, ranging from classical problems in distributed computing, such as assigning computational tasks to parallel processors, to newer applications in multi-agent systems, such as assigning vehicles to charging stations. For more details and applications, we refer the reader to some of the works on the algorithmic version [14, 28, 36], as well as the surveys of Hall [27], Potts and Strusevich [47] and Lenstra et al. [35], and the books of Pinedo [46] and Rinnooy Kan [49].

\subsubsection{The Selfish Scheduling Problem}

The scheduling problem on unrelated selfish machines is the prototypical problem studied by Nisan and Ronen [44] in 1999, when they introduced the field of algorithmic mechanism design. The authors consider the worst-case performance of truthful mechanisms on dominant strategy, truth-telling equilibria, and discover that the well-known Second-Price auction ${ }^{2}$ has an approximation ratio of $n$ for the problem, where $n$ is the number of machines. Despite several attempts over the years, this is still the best-known truthful mechanism. On the other hand, the succession of the best proven lower bounds started with 2 in [44], improved to 2.41 by Christodoulou et al. [9] and to 2.61 by Koutsoupias and Vidali [33], and finally ${ }^{3}$ to 2.75 in the very recent work of Giannakopoulos et al. [24]. Interestingly, Ashlagi et al. [3] showed a matching lower bound of $n$ for anonymous mechanisms (i.e., mechanisms that do not take the identities of the machines into account) and whether there is a better mechanism that is not

\footnotetext{
${ }^{2}$ In the related literature, this mechanism is often referred to as the Vickrey-Clarke-Groves (VCG) mechanism [12, 26, 55]. The mechanism was originally referred to as the "minWork Mechanism" in [44].

${ }^{3}$ During the preparation of our paper, a new manuscript by Dobzinski and Shaulker [15] appeared online, further improving the lower bound to 2.80 .
} 
anonymous is still the most prominent open problem in the area. In any case, anonymity is in general a desirable property which is satisfied by most natural mechanisms (including the best known mechanisms for scheduling [36]); we further discuss the role of this property in our setting in Section 5.

Several other variants of the problem have also been considered over the years, such as randomized mechanisms [37, 40, 44], fractional scheduling [10], Bayesian scheduling [6, 13, 22] or restricted domains where the processing times come from discrete sets [34]. Alongside the approximation ratio results, there has also been work on structural properties and characterizations $[8,16]$. For a more detailed exposition of some of these results, we refer the reader to the survey of Christodoulou and Koutsoupias [7].

\subsubsection{The Truthful Setting vs the Strategic Setting}

As we mentioned earlier, given that truthfulness is a very demanding requirement which imposes strict constraints on the allocation and payment functions, it is an interesting direction to consider whether non-truthful mechanisms could perform better, when evaluated in the worstcase equilibrium. In other words, for a given problem, one could ask the following question:

"Do there exist (non-truthful) mechanisms whose Price of Anarchy outperforms the approximation ratio guarantee of all truthful mechanisms?".

To differentiate, we will refer to the traditional approach of Nisan and Ronen [44] as the truthful setting and to the setting where all mechanisms are explored (with respect to their Nash equilibria) as the strategic setting.

Koutsoupias [31] studied the truthful setting for the problem of unrelated machine scheduling without money but he explicitly advocated the strategic setting as a future direction. This was later pursued in Giannakopoulos et al. [23] for the same problem, where the authors answered the aforementioned question in the affirmative. The same approach was taken in [11] following the results of [18] on the limitations of truthful mechanisms for indivisible item allocation. In the literature of auctions, the strategic setting was studied even in domains for which an optimal truthful mechanism (the VCG mechanism) exists, motivated by the fact that nontruthful mechanisms are being employed in practice, with the Generalized Second-Price auction used by Google for the Adwords allocation being a prominent example [5]. We refer the reader to the survey of Roughgarden et al. [51] for more details.

Somewhat surprisingly, although the exploration of different solution concepts besides dominant strategy equilibria was already explicitly mentioned as a future direction by Nisan and Ronen [44], the strategic setting for the scheduling problem was not studied before our paper. As we mentioned earlier, the answer to the highlighted question above here is negative, but the setting proved out to be quite rich in terms of the achievable trade-off between the two different inefficiency notions.

To the best of our knowledge, ours is the first paper that proposes the systematic study of the trade-off between the Price of Anarchy and the Price of Stability. While preparing our manuscript, we became aware that a trade-off between the two notions was very recently considered also in Ramaswamy et al. [48], though in a fundamentally different setting: the authors of [48] study a special case of covering games, originally introduced by Gairing [20], which is not inherently a mechanism design setup. On the contrary, our interest is in explicitly studying this trade-off in the area of algorithmic mechanism design, thus choosing the prototypical scheduling problem as the starting point.

\section{Model and Notation}

Let $\mathbb{R}_{\geq 0}=[0, \infty)$ denote the nonnegative reals and $\mathbb{N}=\{1,2, \ldots\}$ the positive integers. For any $n \in \mathbb{N}$, let $[n]=\{1,2, \ldots, n\}$. In the strategic scheduling problem (on unrelated machines), 
there is a set $N=\{1, \ldots, n\}$ of machines (or agents) and a set $J=\{1, \ldots, m\}$ of tasks. Each machine $i$ has a processing time (or cost) $t_{i, j} \geq 0$ for task $j$. The induced matrix $\mathbf{t} \in \mathbb{R}_{\geq 0}^{n \times m}$ is the profile of processing times. For convenience, we will denote by $\mathbf{t}_{i}=\left(t_{i, 1}, \ldots, t_{i, m}\right)$ the vector of processing times of machine $i$ for the tasks and by $\mathbf{t}^{j}=\left(t_{1, j}, \ldots, t_{n, j}\right)$ the vector of processing times of the machines for task $j$, so that $\mathbf{t}=\left(\mathbf{t}_{1}, \ldots, \mathbf{t}_{n}\right)=\left(\mathbf{t}^{1}, \ldots, \mathbf{t}^{m}\right)^{\top}$. The machines are strategic and therefore, when asked, they do not necessarily report their true processing times $\mathbf{t}$ but they rather use strategies $\mathbf{s} \in \mathbb{R}_{\geq 0}^{n \times m}$. To emphasize the distinction, we will often refer to $\mathbf{t}$ as the profile of true processing times. Adopting standard game-theoretic notation, we use $\mathbf{t}_{-i}$ and $\mathbf{s}_{-i}$ to denote the profile of true or reported processing times respectively, without the coordinates of the $i$ 'th machine.

A (deterministic, direct revelation) mechanism $\mathcal{M}=(\mathbf{x}, \mathbf{p})$ gets as input a strategy profile $\mathbf{s} \in \mathbb{R}^{n \times m}$ reported by the machines and outputs allocation $\mathbf{x}=\mathbf{x}(\mathbf{s}) \in\{0,1\}^{n \times m}$ and payment $\mathbf{p}=\mathbf{p}(\mathbf{s}) \in \mathbb{R}_{\geq 0}^{n}: x_{i, j}$ is an indicator variable denoting whether or not task $j$ is allocated to machine $j$, and $p_{i}$ is the payment with which $\mathcal{M}$ compensates machine $i$ for taking part in the mechanism. Thus, the allocation rule needs to satisfy $\sum_{i \in N} x_{i, j}(\mathbf{s})=1$ for all tasks $j$.

The utility of machine $i$ under a mechanism $\mathcal{M}=(\mathbf{x}, \mathbf{p})$, given true running times $\mathbf{t}_{i}$ and a reported profile $\mathbf{s}$ by the machines, is

$$
u_{i}^{\mathcal{M}}\left(\mathbf{s} \mid \mathbf{t}_{i}\right)=p_{i}(\mathbf{s})-\sum_{j=1}^{m} x_{i, j}(\mathbf{s}) t_{i, j}
$$

that is, the payment she receives from $\mathcal{M}$ minus the total workload she has to execute. This is exactly the reason why machines may lie about their true processing times; they will change their report $\mathbf{s}_{i}$ and deviate to another $\mathbf{s}_{i}^{\prime}$ if this improves the above quantity. A stable solution with respect to such best-response selfish behaviour is captured by the well-known notion of an equilibrium. Given a mechanism $\mathcal{M}$ and a strategy profile $\mathbf{s}$, we will say that $\mathbf{s}$ is a (pure Nash) equilibrium $^{4}$ of $\mathcal{M}$ (with respect to a true profile $\mathbf{t}$ ) if, for every machine $i$ and every possible deviation $\mathbf{s}_{i}^{\prime} \in \mathbb{R}_{\geq 0}^{m}$,

$$
u_{i}^{\mathcal{M}}(\mathbf{s} \mid \mathbf{t}) \geq u_{i}^{\mathcal{M}}\left(\mathbf{s}_{i}^{\prime}, \mathbf{s}_{-i} \mid \mathbf{t}\right) .
$$

Let $\mathcal{Q}_{\mathbf{t}}^{\mathcal{M}}$ denote the set of pure Nash equilibria of mechanism $\mathcal{M}$ with respect to true profile $\mathbf{t}$. Following the related literature (see, e.g., [11, 23, 44]), we will consider mechanisms for which Nash equilibria exist for every profile of processing times, i.e., $\mathcal{Q}_{\mathbf{t}}^{\mathcal{M}} \neq \emptyset$ for all $\mathbf{t} \in \mathbb{R}_{\geq 0}^{n \times m}$.

Our objective is to design mechanisms that minimize the makespan

$$
C^{\mathcal{M}}(\mathbf{s} \mid \mathbf{t})=\max _{i \in N} \sum_{j=1}^{m} x_{i, j}(\mathbf{s}) t_{i, j},
$$

that is, the total completion time if our machines run in parallel. For a matrix $\mathbf{t}$ of running times, let $\mathrm{OPT}(\mathbf{t})$ denote the optimum makespan, i.e., $\mathrm{OPT}(\mathbf{t})=\min _{\mathbf{y}} \max _{i \in N} \sum_{j=1}^{m} y_{i, j} t_{i, j}$ where $\mathbf{y}$ ranges over all feasible allocation of tasks to machines. It is a well-known phenomenon that equilibria can result in suboptimal solutions, and the following, extensively studied, notions where introduced to quantify exactly this discrepancy: the Price of Anarchy (PoA) and the Price of Stability (PoS) of a scheduling mechanism $\mathcal{M}$ on $n$ machines are, respectively,

$$
\operatorname{PoA}(\mathcal{M})=\sup _{m \in \mathbb{N}, \mathbf{t} \in \mathbb{R}_{\geq 0}^{n \times m}} \frac{\sup _{\mathbf{s} \in \mathcal{Q}_{\mathbf{t}}^{\mathcal{M}}} C^{\mathcal{M}}(\mathbf{s} \mid \mathbf{t})}{\operatorname{OPT}(\mathbf{t})} \quad \operatorname{PoS}(\mathcal{M})=\sup _{m \in \mathbb{N}, \mathbf{t} \in \mathbb{R}_{\geq 0}^{n \times m}} \frac{\inf _{\mathbf{s} \in \mathcal{Q}_{\mathbf{t}}^{\mathcal{M}}} C^{\mathcal{M}}(\mathbf{s} \mid \mathbf{t})}{\operatorname{OPT}(\mathbf{t})} .
$$

For simplicity, we will sometimes drop the $\mathcal{M}, \mathbf{t}$ and $\mathbf{s}$ in the notation introduced in this section, whenever it is clear which mechanism and which true or reported profile we are referring to.

\footnotetext{
${ }^{4}$ We will be interested in pure Nash equilibria in this paper, but we discuss different solution concepts in Section 2.2 as well as in Section 5.
} 


\subsection{Task-Independent Mechanisms}

For a significant part of this paper, we will focus on the class of anonymous, task-independent mechanisms. This is a rather natural class of mechanisms; as a matter of fact, two of the arguably most well-studied and used mechanisms in practice, namely the First-Price and SecondPrice, lie within this class.

Definition 1 (Task-independence). A mechanism $\mathcal{M}=(\mathbf{x}, \mathbf{p})$ is called task-independent if each one of its tasks is allocated independently of the others. Formally, there exists a collection of single-task mechanisms $\left\{\mathcal{A}_{j}\right\}_{j=1, \ldots, m}, \mathcal{A}_{j}=\left(\mathbf{y}^{j}, \mathbf{q}^{j}\right)$, such that, for any task $j$, any machine $i$, and for any strategy profile $\mathbf{s}$,

$$
\mathbf{x}^{j}(\mathbf{s})=\mathbf{y}^{j}\left(\mathbf{s}^{j}\right) \quad \text { and } \quad p_{i}(\mathbf{s})=\sum_{j=1}^{m} q_{i}^{j}\left(\mathbf{s}^{j}\right) .
$$

We will refer to the single-task mechanisms $\mathcal{A}_{j}$ of the above definition as the components of $\mathcal{M}$. It is important to notice here that the definition does not require the mechanism to necessarily use the same component for all the tasks.

Another standard property in the literature of the problem is anonymity. The property can be defined generally (e.g., see $[3,31]$ ), but here we will define it for task-independent mechanisms. Since we are dealing with potentially non-truthful mechanisms, our notion of anonymity needs to refer to the equilibria of the mechanism.

Definition 2 (Anonymity). A single-task mechanism $\mathcal{A}=(\mathbf{x}, \mathbf{p})$ is anonymous if, for any true processing time profile $\mathbf{t}$ with no ties ${ }^{5}$ and any permutation ${ }^{6} \pi$, if there exists an equilibrium $\mathbf{s}$ under $\mathbf{t}$, then there exists an equilibrium $\tilde{\mathbf{s}}$ under true profile $\pi(\mathbf{t})$ with allocation $\mathbf{x}(\tilde{\mathbf{s}})=\pi(\mathbf{x}(\mathbf{s}))$. A task-independent mechanism $\mathcal{M}$ is anonymous, if all its components are anonymous (singletask) mechanisms.

Remark 1. Our notion of anonymity refers to the true profiles, and stipulates that after any permutation of machine identities, a corresponding equilibrium exists. This is the natura analogue of anonymity for the case of Nash equilibria; indeed, if one substitutes the notion of "Nash equilibrium" by "dominant strategy equilibrium" in Definition 2, then the standard notion employed by Ashlagi et al. [3] for truthful mechanisms is recovered. Note that similarly to [3], we only require this property to hold when the profiles of processing times do not exhibit ties.

Perhaps the simplest and most natural mechanism that one can think of is the following, which assigns the task to the fastest machine (according to the declared processing times) and pays her her declaration.

Definition 3 (First-Price (FP) mechanism). Assign each task $j$ to the fastest machine $\iota(j)$ for it, i.e. $\iota(j) \in \arg \min _{i \in N} s_{i, j}$ (breaking ties arbitrarily), paying her her declared running time $s_{\iota(i), j}$; pay the remaining $N \backslash\{\iota(j)\}$ machines 0 for task $j$.

Second-Price mechanisms have also been extensively studied and applied in auction theory, but also in strategic scheduling. As we mentioned in the introduction, the following mechanism is usually referred to as the VCG mechanism in the literature of the problem (see e.g., [7]):

Definition 4 (Second-Price (SP) mechanism). Assign each task $j$ to the fastest machine $\iota(j)$ for it, i.e., $\iota(j) \in \arg \min _{i \in N} s_{i, j}$ (breaking ties arbitrarily), paying her the declared processing time of the second-fastest machine, i.e. $\min _{i \in N \backslash\{\iota(j)\}} s_{i, j}$; pay the remaining $N \backslash\{\iota(j)\}$ machines 0 for task $j$.

\footnotetext{
${ }^{5}$ That is, $t_{i} \neq t_{i^{\prime}}$ for all $1 \leq i \neq i^{\prime} \leq n$.

${ }^{6}$ For any permutation $\pi:\{1, \ldots, n\} \longrightarrow\{1, \ldots, n\}$ and $n$-dimensional vector $\mathbf{x}=\left(x_{1}, \ldots, x_{n}\right)$, the permutation of $\mathbf{x}$ under $\pi$ is the vector $\pi(\mathbf{x}) \equiv\left(x_{\pi(1)}, \ldots, x_{\pi(n)}\right)$.
} 


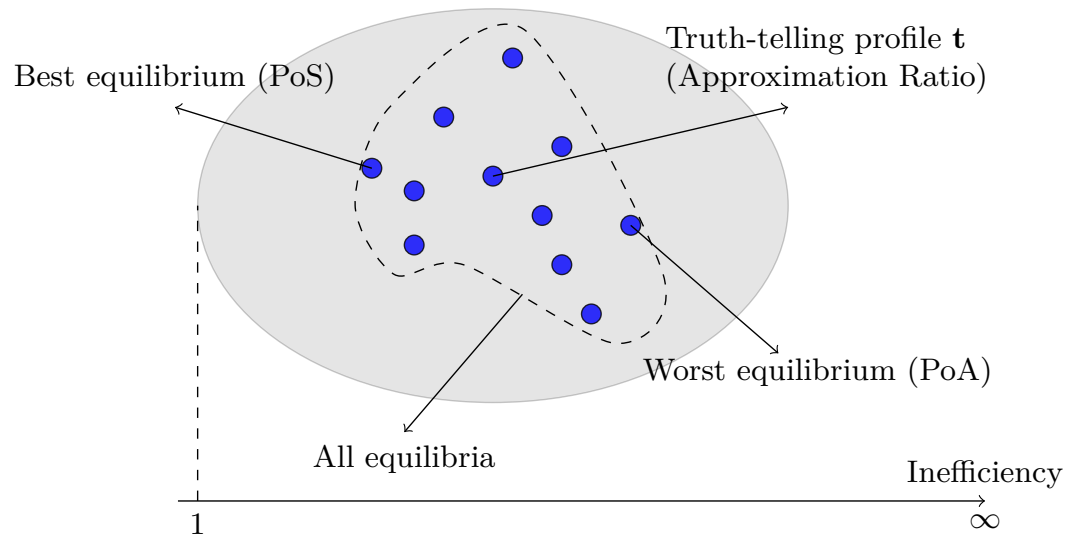

Figure 1: A pictorial representation of the relation between the different solution concepts and the notions of inefficiency. The blue nodes represent the set of equilibria of a mechanism $\mathcal{M}$ for a fixed true underlying profile of processing times $\mathbf{t}$ (they are depicted as a finite set, for convenience, but this need not be the case). The wider grey area is the set of all feasible input strategy profiles $\mathbf{s}$ of $\mathcal{M}$. We have marked the best and worst (under $\mathbf{t}$ ) equilibria (assuming they are unique, for ease of exposition), as well as the truth-telling profile. Note that if $\mathcal{M}$ is truthful, then the truth-telling profile is a (dominant strategy) equilibrium (but, in general, this profile might not even belong to the set of equilibria). The PoA bounds the inefficiency of all the blue nodes, the PoS bounds the inefficiency of the left-most node, and the approximation ratio bounds the inefficiency of the truth-telling node; all these bounds are computed, in the worst case, over all possible true profiles t).

Notice that both FP and SP mechanisms are task-independent and anonymous. Furthermore, SP is truthful. As a matter of fact, SP is the best known truthful mechanism if one is interested only in dominant strategy equilibria (see, e.g., [9, 44] and Section 2.2).

\subsection{Solution Concepts and Notions of Inefficiency}

The solution concept that we consider in this paper is that of the pure Nash equilibrium. In the literature of the truthful scheduling problem, the employed solution concept is that of the dominant strategy equilibrium, i.e., a strategy profile in which no agent would have an incentive to deviate to any other strategy, no matter the strategies of the remaining agents. More precisely, the literature has been interested in truthful mechanisms, i.e., mechanisms for which truth-telling is always (i.e., for any processing time profile t) a dominant strategy equilibrium. The goal is to find a mechanism with the best approximation ratio, which is defined as the worst-case (over all inputs) ratio of the makespan of the mechanism over the optimal makespan, in the truth-telling equilibrium.

For this objective, studying only the truth-telling dominant strategy equilibria is without loss of generality, by the Revelation Principle (see, e.g., [43]). We remark however that, a priori, the fact that an allocation function can be implemented in truth-telling dominant strategies (i.e., an appropriate payment function can be found such that the resulting mechanism is truthful) does not have any implications on the space of non-truthful mechanisms and their PoA/PoS guarantees.

There are however some inherent relations between the approximation ratio, the Price of Anarchy and the Price of Stability which follow directly from their definitions. Clearly, a Price of Anarchy guarantee is stronger than a Price of Stability guarantee, since the former bounds the inefficiency of all equilibria while the latter is only concerned with the best one. Since dominant strategy equilibria are also Nash equilibria by definition, for truthful mechanisms, a Price of Anarchy guarantee is also stronger than an approximation ratio guarantee, which, in turn, is stronger than a Price of Stability guarantee. An illustration of the relation between these different notions is given in Fig. 1. 


\section{The Inefficiency of All Mechanisms}

We start with a lower bound of $n$ for the Price of Anarchy of the scheduling problem, which applies to all mechanisms. The lower bound will be based on the following monotonicity lemma. We note that this monotonicity property is different from the weak monotonicity (WMON) used in the literature of truthful machine scheduling (see e.g., $[9,44]$ ), in the sense that (a) it is global, whereas WMON is local and (b) it applies to the relation between the true processing times and the equilibria of the mechanism, rather than the actual allocations.

Lemma 1 (Equilibrium Monotonicity). Let $\mathcal{M}$ be any mechanism for the scheduling problem. Let $\mathbf{t}$ be a profile of true processing times and let $\mathbf{s} \in \mathcal{Q}_{\mathbf{t}}$ be an equilibrium under $\mathbf{t}$. Denote by $S_{i}$ the set of tasks assigned to machine $i$ by $\mathcal{M}$ on input $\mathbf{s}$. Consider any profile $\hat{\mathbf{t}}$ such that for every machine $i, \hat{t}_{i, j} \leq t_{i, j}$ if $j \in S_{i}$ and $\hat{t}_{i, j} \geq t_{i, j}$ if $j \notin S_{i}$. Then $\mathbf{s} \in \mathcal{Q}_{\hat{\mathbf{t}}}$, i.e., $\mathbf{s}$ is an equilibrium under $\hat{\mathbf{t}}$ as well.

Proof. Assume by contradiction that $\mathbf{s} \notin \mathcal{Q}_{\hat{\mathbf{t}}}$, which means that for the profile of processing times $\hat{\mathbf{t}}$, there exists some machine $i$ that has a beneficial deviation $\mathbf{s}_{i}^{\prime}$, i.e., $u_{i}\left(\mathbf{s}_{i}^{\prime}, \mathbf{s}_{-i} \mid \hat{\mathbf{t}}\right)>u_{i}(\mathbf{s} \mid \hat{\mathbf{t}})$. Let $S_{i}^{\prime}$ be the set of tasks assigned to machine $i$ under report $\mathbf{s}^{\prime}=\left(\mathbf{s}_{i}^{\prime}, \mathbf{s}_{-i}\right)$ (and underlying true reports $\hat{\mathbf{t}}$ ). The difference in utility for machine $i$ between profiles $\mathbf{s}^{\prime}$ and $\mathbf{s}$ is

$$
\Delta u_{i}(\hat{\mathbf{t}}) \equiv u_{i}\left(\mathbf{s}^{\prime} \mid \hat{\mathbf{t}}\right)-u_{i}(\mathbf{s} \mid \hat{\mathbf{t}})=p_{i}\left(\mathbf{s}^{\prime}\right)-p_{i}(\mathbf{s})+\sum_{j \in S_{i} \backslash S_{i}^{\prime}} \hat{t}_{i, j}-\sum_{j \in S_{i}^{\prime} \backslash S_{i}} \hat{t}_{i, j}
$$

By the fact that $s_{i}^{\prime}$ is a beneficial deviation, it holds that $\Delta u_{i}(\hat{\mathbf{t}})>0$.

Now consider the profile of processing times $\mathbf{t}$ and the same deviation $\mathbf{s}_{i}^{\prime}$ of machine $i$. The increase in utility is

$\Delta u_{i}(\mathbf{t})=p_{i}\left(\mathbf{s}^{\prime}\right)-p_{i}(\mathbf{s})+\sum_{j \in S_{i} \backslash S_{i}^{\prime}} t_{i, j}-\sum_{j \in S_{i}^{\prime} \backslash S_{i}} t_{i, j} \geq p_{i}\left(\mathbf{s}^{\prime}\right)-p_{i}(\mathbf{s})+\sum_{j \in S_{i} \backslash S_{i}^{\prime}} \hat{t}_{i, j}-\sum_{j \in S_{i}^{\prime} \backslash S_{i}} \hat{t}_{i, j}=\Delta u_{i}(\hat{\mathbf{t}})$,

which holds because $t_{i, j} \geq \hat{t}_{i, j}$, if $j \in S_{i}$ and $t_{i, j} \leq \hat{t}_{i, j}$, if $j \notin S_{i}$. This implies that $\Delta u_{i}(\mathbf{t})>0$, which contradicts the fact that $\mathbf{s} \in \mathcal{Q}_{\mathbf{t}}$.

Using this lemma, we can prove our first lower bound:

Theorem 1. For any scheduling mechanism $\mathcal{M}$ for $n$ machines, it must be that $\operatorname{PoA}(\mathcal{M}) \geq n$.

Proof. Let $\mathcal{M}$ be any mechanism and consider a profile of true processing times $\mathbf{t}$ with $n$ machines and $n^{2}$ tasks, where $t_{i, j}=1$ for all machines $i$ and all tasks $j$. Let $\mathbf{s}=\left(s_{1}, s_{2}, \ldots, s_{n}\right)$ be a pure Nash equilibrium of $\mathcal{M}$ under $\mathbf{t}$. For each machine $i$, let $S_{i}$ be the set of tasks assigned to that machine and note that there exists some machine $k$ for which $\left|S_{k}\right| \geq n$. Let $T_{k} \subseteq S_{k}$ be any subset of $S_{k}$ such that $\left|T_{k}\right|=n$.

Now consider the following profile $\hat{t}$ of processing times:

- For all $i \neq k, \hat{t}_{i, j}=0$, for all $j \in S_{i}$ and $\hat{t}_{i, j}=t_{i, j}$, for all $j \notin S_{i}$.

- $\hat{t}_{k j}=0$, for all $j \in S_{k} \backslash T_{k}$ and $\hat{t}_{k j}=t_{k, j}$, for all $j \notin S_{k} \backslash T_{k}$.

By Lemma 1 , the profile $\mathbf{s}=\left(s_{1}, s_{2}, \ldots, s_{n}\right)$ is a pure Nash equilibrium under $\hat{\mathbf{t}}$ and the allocation is the same as before, for a makespan of at least $n$, since machine $k$ is assigned all the tasks in $T_{k}$. The optimal allocation will assign one task from $T_{k}$ to each machine, the tasks from $S_{i}$ to machine $i$ for each $i \neq k$ and the tasks from $S_{k} \backslash T_{k}$ to machine $k$, for a total makespan of 1 and the Price of Anarchy bound follows. 


\subsection{PoA/PoS Trade-off}

In this section, we prove our main theorem regarding the trade-off between the Price of Anarchy and the Price of Stability. The theorem informally says that if the Price of Anarchy of a mechanism is small, then its Price of Stability has to be high.

Theorem 2. For any scheduling mechanism $\mathcal{M}$ for $n$ machines, and any positive real $\alpha$,

$$
\operatorname{PoA}(\mathcal{M})<\alpha \quad \Longrightarrow \quad \operatorname{PoS}(\mathcal{M}) \geq \frac{n-1}{\alpha}+1
$$

Proof. By performing the transformation $\rho=\frac{n-1}{\alpha}+1$ and taking the contrapositive, it is not difficult to see that we need to prove that

$$
\operatorname{PoS}(\mathcal{M})<\rho \quad \Longrightarrow \quad \operatorname{PoA}(\mathcal{M}) \geq \frac{n-1}{\rho-1},
$$

for any real $\rho>1$.

Consider an instance with $n$ agents and $n$ tasks. Assume a true $n \times n$ processing-times matrix $\mathbf{t}$ with

$$
t_{1, j}= \begin{cases}n-1, & \text { if } j=1 \\ \rho-1, & \text { otherwise }\end{cases}
$$

and

$$
t_{i, j}= \begin{cases}n-1, & \text { if } j=i, \\ \infty, & \text { otherwise }\end{cases}
$$

for all $i=2, \ldots, n$. Here $\infty$ denotes an arbitrarily large positive value, and actually replacing it with any value $M \geq \rho(n-1)$ will work just fine for our proof. ${ }^{7}$

First notice that by allocating each task $j$ to machine $j$ with running time $t_{j, j}=n-1$, for all $j \in[n]$, we get an upper bound of $n-1$ on the optimal makespan of $\mathbf{t}$. Thus, since $\operatorname{PoS}(\mathcal{M})<\rho$, there must exist a pure Nash equilibrium profile $\mathbf{s}^{\star}$ such that the allocation $\mathcal{M}\left(\mathbf{s}^{\star}\right)$ results in a makespan less than $\rho(n-1)$ (with respect to the underlying, true time matrix $\mathbf{t})$. But then, due to the structure of $\mathbf{t}$, and in particular the large value of $M, \mathcal{M}\left(\mathbf{s}^{\star}\right)$ can only allocate each task $j$ to either machine 1 or machine $j$, for all $j \in[n]$. In particular, task 1 will necessarily have to be allocated to machine 1 . Furthermore, from the remaining $n-1$ tasks, not all of them can be allocated to machine 1 , because that would give rise to a running time of $t_{1,1}+\sum_{j=2}^{n} t_{1, j}=n-1+(n-1)(\rho-1)=\rho(n-1)$ for machine 1 , which violates the Price of Stability constraint assumed for $\mathbf{s}^{\star}$. So, there must exist at least one task $j \geq 2$, denote it by $j^{\star}$, such that $\mathcal{M}\left(\mathbf{s}^{\star}\right)$ allocates $j$ to machine $j$.

For each task $j$, let $i_{j}$ denote the machine which task $j$ is allocated to by $\mathcal{M}\left(\mathbf{s}^{\star}\right)$. Now modify the original, true execution time matrix $\mathbf{t}$ by changing the running time $t_{i_{j}, j}$, for all $j \neq j^{\star}$, to $t_{i_{j}, j}^{\prime}=0$. Denote this new matrix by $\mathbf{t}^{\prime}$. Due to Lemma $1, \mathbf{s}^{\star}$ has to be a pure Nash equilibrium of $\mathcal{M}$ with respect to the modified true profile $\mathbf{t}^{\prime}$ as well. But now $\mathcal{M}\left(\mathbf{s}^{\star}\right)$ results in a makespan of at least $t_{j^{\star}, j^{\star}}^{\prime}=t_{j^{\star}, j^{\star}}=n-1$ (since task $j^{\star}$ is allocated to machine $j^{\star}$ ), while allocating $j^{\star}$ to machine 1 (and leaving all other assignments as they are, i.e. task $j \neq j^{\star}$ gets allocated to machine $\left.i_{j}\right)$ results in machine 1 having a total running cost of at most $(n-1) \cdot 0+t_{1, j^{\star}}^{\prime}=\rho-1$, and all other machines 0. This gives a Price of Anarchy lower bound of $\frac{n-1}{\rho-1}$.

By allowing $\alpha$ in Theorem 2 to grow arbitrarily large, we get the following:

Corollary 1. Even for just two machines, if a scheduling mechanism has an optimal Price of Stability of 1, then its Price of Anarchy has to be unboundedly large.

\footnotetext{
${ }^{7}$ We will adopt a similar convention throughout the paper.
} 


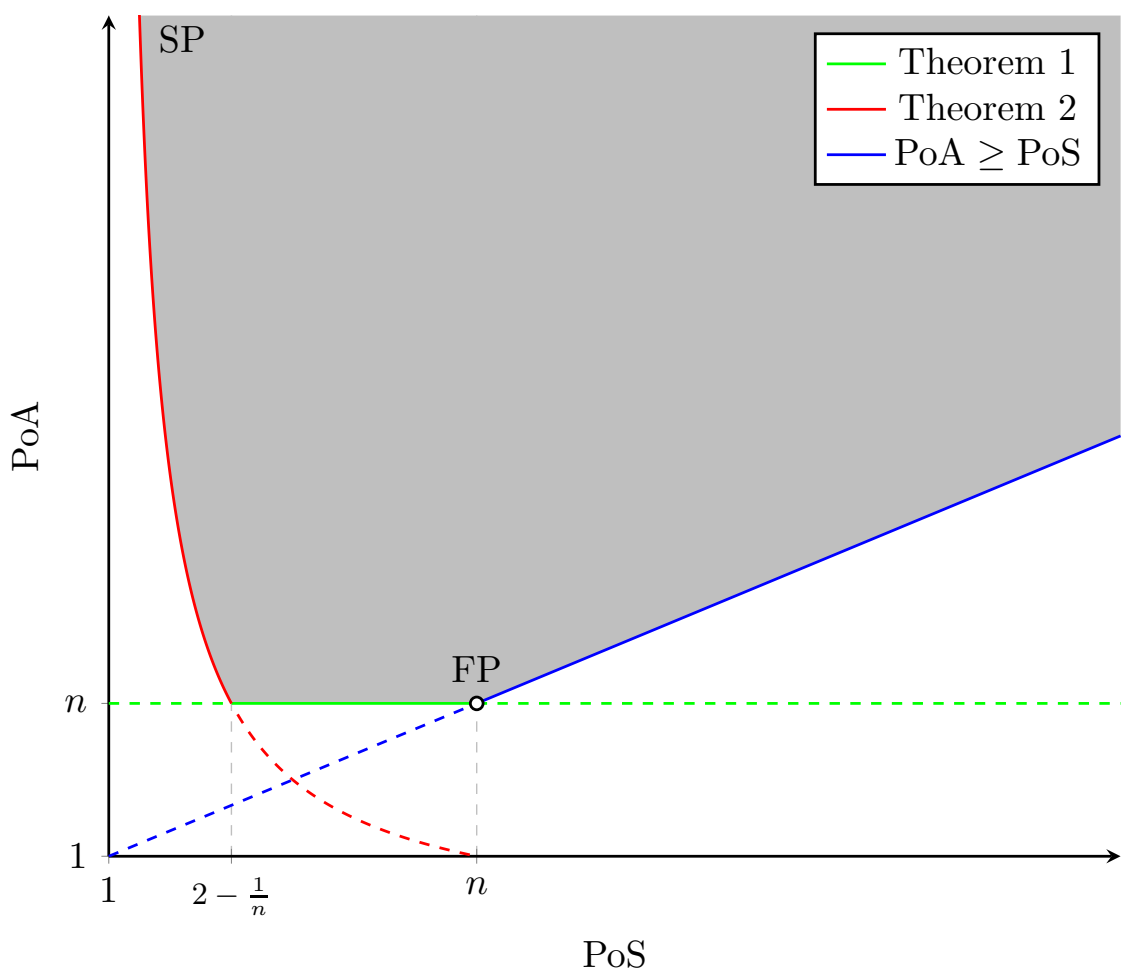

Figure 2: The inefficiency boundary for general mechanisms, given by Theorem 2 (red line). Combined with the global PoA lower bound of Theorem 1 (green line) and the trivial fact that the PoS is at most the PoA (blue line), we finally get the grey feasible region.

From the results of this section, as well as the trivial fact that $\operatorname{PoA}(\mathcal{M}) \geq \operatorname{PoS}(\mathcal{M})$ for any mechanism $\mathcal{M}$, we obtain a feasibility trade-off between the PoA and the PoS of scheduling mechanisms, which is illustrated in Fig. 2. We refer to the boundary of the shaded feasible region as the inefficiency boundary; the shape of the boundary follows from Theorem 2, as well as Theorem 1, since for $\operatorname{PoS}(\mathcal{M})>2-\frac{1}{n}$ (or, in the language of Theorem 2, for $\alpha<n$ ), the best (i.e. largest) lower bound on the PoA is now given by Theorem 1 .

\subsubsection{Mechanisms on the Extrema of the Inefficiency Boundary}

When looking for mechanisms on the Pareto frontier, the first ones that come to mind are perhaps the First-Price (FP) and Second-Price (SP) mechanisms, defined in Section 2, which are straightforward adaptations of the well-known First-Price auction and Second-Price auction mechanisms from the auction literature.

It follows from known results in the literature for the First-Price auction (see, e.g., [17]) that in every pure Nash equilibrium of the FP, each task is allocated to the machine with the smallest true processing time for the task; we provide a simple proof below for completeness. In Section 4.2, we will define a class of task-independent mechanisms $\left(\mathcal{S P} \mathcal{P}_{\alpha}\right)$ that contain $\mathrm{FP}$ as a corner case $\left(\mathcal{S P} \mathcal{P}_{1}\right)$.

Theorem 3. The PoA and the PoS of the First-Price mechanism are both $n$.

Proof. First, we argue that in every equilibrium of FP, for any task $j$ a machine with the fastest true processing time for $j$ receives the task. Given any profile of true processing times $\mathbf{t}$, let $J_{a}$ be the set of machines with the fastest true processing time $t_{f}$ for task $j$. Assume by contradiction that some machine $k \notin J_{a}$ receives the task at some equilibrium $\mathbf{s}^{j}$. Since $\mathbf{s}^{j}$ is an equilibrium, it must be the case that $p_{k, j}=s_{k, j} \geq t_{k, j}>t_{f}$, as otherwise machine $k$ would have negative utility. But then, by the continuity of the strategy space, any machine $i \in J_{a}$ 
can report $s_{i, j}^{\prime} \in\left(t_{f}, s_{k, j}\right)$ and win the task, obtaining positive utility. This contradicts the fact that $\mathbf{s}^{j}$ is an equilibrium.

Given this, it is not hard to see that $\mathrm{PoA}(\mathrm{FP})=n$, as in the worst-case, every task will go to the same machine, which will be the fastest machine for all tasks. To show that $\operatorname{PoS}(\mathrm{FP})=n$, it is easy to construct an example where there is a single machine $k$ that has the fastest processing time $t_{f}$ for each task, and $n-1$ different machines $i_{1}, \ldots, i_{n-1}$, such that machine $i_{j}$ has processing time $t_{f}+\varepsilon$ for task $j$, where $\varepsilon$ can be arbitrarily small. In every equilibrium, all tasks go to machine $k$ for a makespan of $n \cdot t_{f}$, whereas in the optimal schedule, machine $k$ receives task 1 and machine $i_{j}$ receives task $j$. The Price of Stability goes to $n$ as $\varepsilon \rightarrow 0$.

For the Second-Price mechanism, again it follows from known observations in the literature that while the mechanism is truthful, it has several other pure Nash equilibria as well. More precisely, for a task $j$ and any machine $i$, there exists an equilibrium for which task $j$ is allocated to machine $i$. Therefore, we have the following:

Theorem 4. The PoA of the Second-Price mechanism is unbounded and its $\mathrm{PoS}$ is 1.

Theorem 4 can be obtained as a corollary of our results in Section 4.2, since SP is also a corner-case mechanism in our class, namely $\mathcal{S P} \mathcal{D}_{\infty}$. Interestingly, as we identify in Theorem 5 below, it turns out that the bad PoA bound is a inherent characteristic of all truthful mechanisms. In other words, if one is interested in the set of all equilibria, they would have to reach out beyond truthful mechanisms.

Theorem 5. The Price of Anarchy of any truthful mechanism is unbounded.

Proof. Let $\mathcal{M}=(\mathbf{x}, \mathbf{p})$ be a truthful mechanism on $n \geq 2$ machines. To arrive to a contradiction, assume that there exists a real $M \geq 1$ such that $\operatorname{PoA}(\mathcal{M}) \leq M$. We will consider singletask instances with only $n=2$ machines. This is without loss of generality, since one can add arbitrarily many more machines with 0 running time for the task, and the proof remains valid. In particular, we assume an underlying vector of true running times $\mathbf{t}=(0, \varepsilon)$, where $\varepsilon \in\left(0, M^{-1}\right)$, and a vector $\mathbf{s}=(1,0)$ of reported costs. We first show that $\mathcal{M}$ allocates the task to machine $i=2$, even if she reports slightly slower running times.

Claim 1. For any $\delta \in[0, \varepsilon]$, mechanism $\mathcal{M}$ always allocates the task to machine $i=2$ on any input $\mathbf{s}^{\prime}=(1, \delta)$.

Proof. First notice that, due to truthfulness, if we consider as true underlying profile $\mathbf{t}^{\prime}=\mathbf{s}^{\prime}=$ $(1, \delta)$, then $\mathbf{s}^{\prime}$ has to be an equilibrium. Next, for a contradiction, assume that there exists a nonnegative $\delta \leq \varepsilon$ such that $x_{2}\left(\mathbf{s}^{\prime}\right)=0$. Then $x_{1}\left(\mathbf{s}^{\prime}\right)=1$, and thus the makespan under equilibrium $\mathbf{s}^{\prime}$ (and true profile $\mathbf{t}^{\prime}$ ) would be 1, while an optimal solution would have given the task to the faster machine, for a makespan of $\delta$. This results in a PoA of at least $\frac{1}{\delta} \geq \varepsilon^{-1}>M$ which is a contradiction. This completes the proof of the claim.

Using the same argument, we can also show that $\mathcal{M}$ keeps allocating the task to machine $i=2$ as long as the other machine has a strictly positive cost:

Claim 2. For any $\delta>0$, mechanism $\mathcal{M}$ always allocates the task to machine $i=2$ on any input $\mathbf{s}^{\prime}=(\delta, 0)$.

We are now ready to prove that $\mathbf{s}$ is actually an equilibrium:

Claim 3. Reporting $\mathbf{s}=(1,0)$ is an equilibrium of $\mathcal{M}$ (under true costs $\mathbf{t}$ ).

The above claim is enough to complete the proof, since by combining it with the previous Claim 1 (with $\delta=0$ ) we get that the makespan of $\mathcal{M}$ under equilibrium $\mathbf{s}$ is $\varepsilon>0$ (since the task goes to machine $i=2$ ) while the optimal one is 0 . This contradicts the fact that $\operatorname{PoA}(\mathcal{M})$ is bounded. 
Proof of Claim 3. First we remark that, due to well-known characterizations of truthfulness for single-dimensional domains [41] (which apply to our case, since we have a single task), there exist real functions $h_{1}, h_{2}$ such that the utilities of our machines (with respect to true costs $\mathbf{t}$ ) on any vector of reports $\mathbf{s}^{\prime}$ are given by ${ }^{8}$

$$
u_{i}\left(\mathbf{s}^{\prime}\right)=h_{i}\left(s_{-i}^{\prime}\right)+\left(s_{i}^{\prime}-t_{i}\right) \cdot x_{i}\left(\mathbf{s}^{\prime}\right)-\int_{0}^{s_{i}^{\prime}} x_{i}\left(z, s_{-i}^{\prime}\right) d z .
$$

Furthermore, the allocation function $x_{i}\left(\mathbf{s}^{\prime}\right)$ of each agent $i$ is monotonically nonincreasing with respect to her reported cost $s_{i}^{\prime}$.

Now we show that the first agent has no incentive to deviate from reporting $s_{1}=1$ as long as the second agent is fixed at $s_{2}=0$. Indeed, currently, and as long as she reports any strictly positive cost $s_{1}^{\prime}=z>0$, she will still lose the task (i.e., $x_{1}\left(z, s_{2}\right)=0$ ). That holds due to Claim 2. From (1), this results in a utility of $u_{1}\left(s_{1}^{\prime}, s_{2}\right)=h_{1}\left(s_{2}\right)$. For the only remaining case that she reports $s_{1}=0^{\prime}$, again from (1) we get that $u_{1}\left(0, s_{2}\right)=h_{1}\left(s_{2}\right)-(0-0) \cdot x_{1}\left(0, s_{2}\right)=h_{1}\left(s_{2}\right)$. Thus, in no case the first agent can gain by unilaterally deviating.

Finally, we need to show that the second agent has no incentive to deviate from reporting $s_{2}=0$ as well. Assuming the other agent fixed at $s_{1}=1$, the improvement in her utility by declaring a cost $s_{2}^{\prime} \geq 0$ is (due to (1))

$$
u_{2}\left(1, s_{2}^{\prime}\right)-u_{2}(1,0)=\left(s_{2}^{\prime}-\varepsilon\right) x_{2}\left(1, s_{2}^{\prime}\right)-\int_{0}^{s_{2}^{\prime}} x_{2}(1, z) d z .
$$

Recall now from Claim 1 that $x_{1}(1, z)=1$ for all $z \in[0, \varepsilon]$. Thus, for $s_{2}^{\prime} \in[0, \varepsilon]$ the above difference in the second agent's utility becomes $\left(s_{2}^{\prime}-\varepsilon\right)-s_{2}^{\prime}=-\varepsilon<0$, while for $s_{2}^{\prime}>\varepsilon$ it is

$$
\left(s_{2}^{\prime}-\varepsilon\right) x_{2}\left(1, s_{2}^{\prime}\right)-\int_{\varepsilon}^{s_{2}^{\prime}} x_{2}(1, z) d z \leq\left(s_{2}^{\prime}-\varepsilon\right) x_{2}\left(1, s_{2}^{\prime}\right)-\left(s_{2}^{\prime}-\varepsilon\right) x_{2}\left(1, s_{2}^{\prime}\right) x_{2}\left(1, s_{2}^{\prime}\right)=0,
$$

the inequality holding due to the fact that $x_{2}(1, z)$ is nonincreasing with respect to $z$.

From Theorem 2, Theorem 3 and Theorem 4, it is clear that both FP and SP lie on the boundary of the PoA/PoS feasibility space (see Fig. 2).

\section{The Pareto Frontier of Task-Independent Mechanisms}

As we noted in the previous section, both the SP and FP mechanisms, which lie on the inefficiency boundary (see Fig. 2), are anonymous task-independent mechanisms. In this section, we will construct a tighter boundary on the PoA/PoS trade-off for the class of anonymous task-independent mechanisms. Furthermore, we will show that this boundary is actually tight, by designing a class of optimal mechanisms that lie exactly on it, meaning that for each point on the boundary, there is a mechanism in our class that achieves the corresponding PoA/PoS trade-off. Thus, this results in a complete characterization of the Pareto frontier between the PoA and the PoS. ${ }^{9}$ For an illustration, see Fig. 3.

\footnotetext{
${ }^{8}$ See $[2$, Theorem 4.2$]$.

${ }^{9}$ To prevent any potential confusion, we use the term "inefficiency boundary" to refer to the boundary of the feasible region for the PoA/PoS trade-off, that is defined by some impossibility-type result such as Theorem 6 and we reserve the term "Pareto frontier" for a boundary that can provably not be improved, since there are mechanisms that achieve the corresponding trade-offs. Intuitively, in our terminology, the inefficiency boundary is a "bound" on the achievable Pareto frontier.
} 


\subsection{PoA/PoS Trade-off}

We start with the theorem that gives us the improved boundary on the space of feasible taskindependent and anonymous mechanisms. This is the red line in Fig. 3. Intuitively, the proof of this theorem is based on the following idea: consider two alternative true cost matrices,

$$
\left(\begin{array}{ccccc}
1 & \infty & \cdots & \infty & \infty \\
\infty & 1 & \ddots & \infty & \infty \\
\infty & \infty & \ddots & \ddots & \vdots \\
\vdots & \vdots & \ddots & 1 & \infty \\
\infty & \infty & \ddots & \infty & 1 \\
\alpha^{*} & \alpha^{*} & \cdots & \alpha^{*} & \alpha^{*}
\end{array}\right) \quad \text { and } \quad\left(\begin{array}{ccccc}
\alpha & \infty & \cdots & \infty & \infty \\
\infty & \alpha & \ddots & \infty & \infty \\
\infty & \infty & \ddots & \ddots & \vdots \\
\vdots & \vdots & \ddots & \alpha & \infty \\
\infty & \infty & \ddots & \infty & \alpha \\
1^{*} & 1^{*} & \cdots & 1^{*} & 1^{*}
\end{array}\right)
$$

where $\alpha>1$. Then, any anonymous mechanism would either (a) have some equilibrium where all tasks get allocated to the "slow" machine (with running time $\alpha$ ) on the first instance, or (b) at all equilibria it would need to allocate all tasks to the "fast" machine (with running time 1) on the second instance. Case (a) would result in a "high" PoA, while case (b) in a "high" PoS.

Theorem 6. For any task-independent anonymous scheduling mechanism $\mathcal{M}$ for $n$ machines, and any real $\alpha>1$,

$$
\operatorname{PoA}(\mathcal{M})<(n-1) \alpha+1 \quad \Longrightarrow \quad \operatorname{PoS}(\mathcal{M}) \geq \frac{(n-1)}{\alpha}+1 .
$$

Proof. Fix a mechanism $\mathcal{M}$ on $n \geq 2$ machines and $m=\max \{2,2 n-3\}$ tasks, that allocates each task $j$ independently by running an anonymous single-task mechanism $\mathcal{A}_{j}$. Each such mechanism $\mathcal{A}_{j}$ takes as input a declared cost vector $\mathbf{s}^{j}=\left(s_{1, j}, \ldots, s_{n, j}\right)$ by the machines, where $s_{i, j}$ is the report of machine $i$ for task $j, i=1, \ldots, n$. Besides the reports, recall that there is also an underlying true cost vector $\mathbf{t}^{j}=\left(t_{1, j}, \ldots, t_{n, j}\right)$. Also, fix a parameter $\alpha>1$ and assume that $\operatorname{PoA}(\mathcal{M})<(n-1) \alpha+1$.

We are particularly interested in true cost vectors that have a specific structure, namely being permutations of $(1, \alpha, \infty, \ldots, \infty)$. We will call such cost vectors canonical for the remainder of this proof. Formally, $\mathbf{t}^{j}$ is canonical if:

- there is a unique machine $i^{\prime}$ such that $t_{i^{\prime}, j}=1$ (machine $i^{\prime}$ will be called fast for task $j$ ),

- there is a unique machine $i^{\prime \prime} \neq i^{\prime}$ such that $t_{i^{\prime \prime}, j}=\alpha$ (machine $n$ will be called slow for task $j$ ),

- all other machines $i \neq i^{\prime}, i^{\prime \prime}$ have arbitrarily high, pairwise distinct, processing times for task $j$, that for simplicity we'll denote with $t_{i, j}=\infty$ (these machines will be called dummy).

Notice that, since $\mathcal{M}$ has a bounded PoA, none of its component mechanisms $\mathcal{A}_{j}$ can have an equilibrium, ${ }^{10}$ on any true canonical cost vector $\mathbf{t}^{j}$, that allocates task $j$ to a dummy machine.

The following definition will be helpful for our exposition in the rest of the proof:

Definition 5 (Well-behaved tasks). A task $j$ will be called well-behaved if, for all true canonical cost vectors $\mathbf{t}^{j}$, mechanism $\mathcal{A}_{j}$ allocates task $j$ to the fast machine on all equilibria $\mathbf{s}^{j}$.

\footnotetext{
${ }^{10}$ In multiple points throughout this proof we will silently be using the fact that a profile of reports $\mathbf{s}$ is an equilibrium of $\mathcal{M}$ with respect to a true profile $\mathbf{t}$, if and only if, for all tasks $j, \mathbf{s}^{j}$ is an equilibrium of $\mathcal{A}_{j}$ with respect to $\mathbf{t}^{j}$; this is an immediate consequence of task-independence (see Definition 1 ).
} 


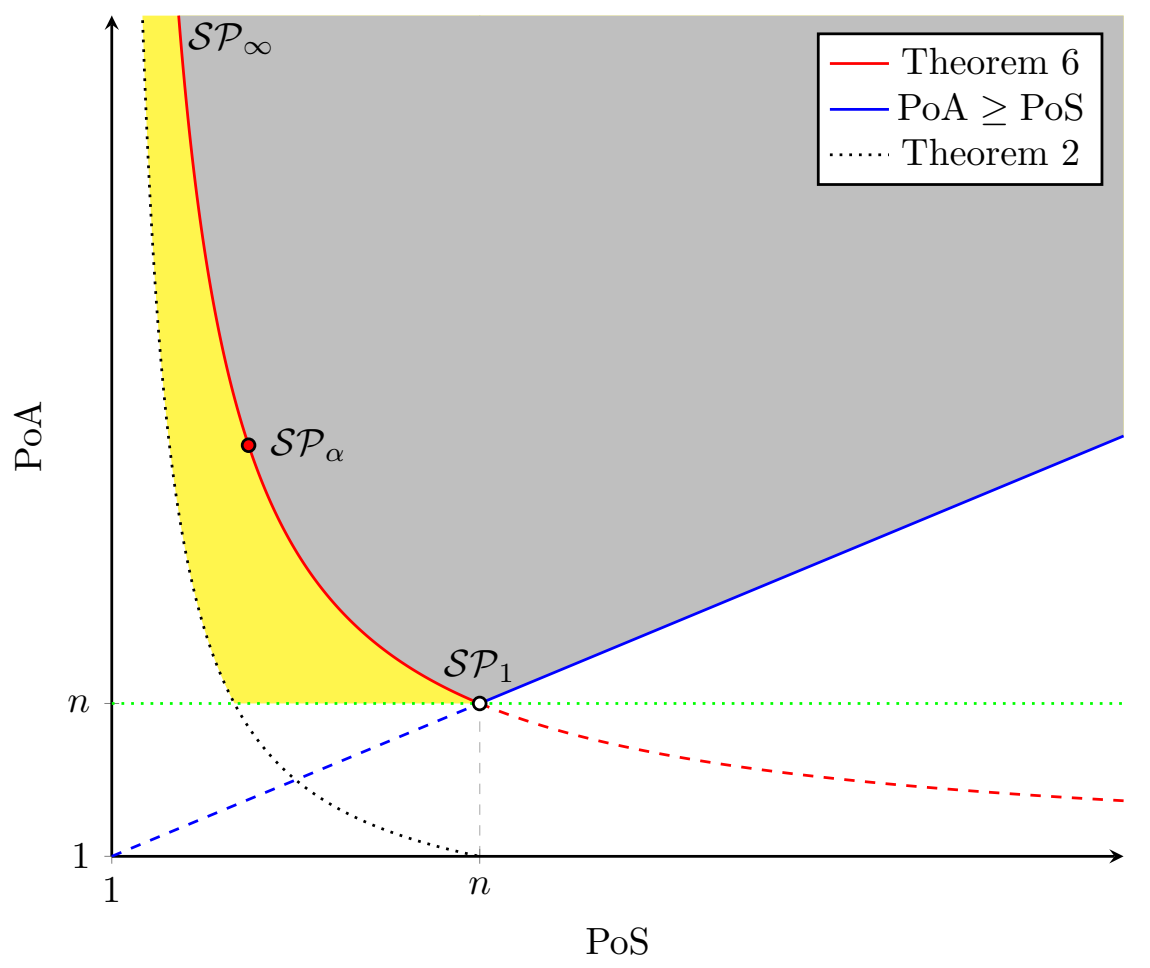

Figure 3: The inefficiency boundary, for anonymous task-independent mechanisms, given by Theorem 6 (red line). Combined with the global PoA lower bound of Theorem 1 (green line) and the trivial fact that the PoS is at most the PoA (blue line), we finally get the grey feasible region. The family of mechanisms $\mathcal{S P}_{\alpha}$ described in Section 4.2 lies exactly on this boundary (red line), thus completely characterizing the Pareto frontier in a smooth way with respect to parameter $\alpha \geq 1$ : on its one end $(\alpha=1)$ is the First-Price mechanism FP $=\mathcal{S P}_{1}$ and at the other $(\alpha \rightarrow \infty)$ the Second-Price mechanism SP $=\mathcal{S P} \mathcal{P}_{\infty}$. The yellow region represents the possible trade-off between the Price of Anarchy and the Price of Stability for mechanisms that are not anonymous or task-independent, as defined by the right boundary in this figure, and the left boundary of Fig. 2 (shown as a red line there). We remark that the "thickness" of this region is mainly due to mechanisms which are not task-independent; as we show in Theorem 9, the corresponding boundary line for mechanisms which are taskindependent but not anonymous is much closer to the red line in the figure.

It turns out that, due to anonymity, a much weaker condition is actually enough in order to establish that a task is well-behaved:

Claim 4. A task is well-behaved if there exists a true canonical cost vector with respect to which all equilibria assign it to the fast machine.

Proof. Assume that there exists a canonical cost vector $\mathbf{t}^{j}$ such that, for all equilibria $\mathbf{s}^{j}$ of $\mathbf{t}^{j}$, mechanism $\mathcal{A}_{j}$ assigns task $j$ to its fast machine. For a contradiction, assume that there also exists a canonical cost vector $\tilde{\mathbf{t}}^{j}$ and an equilibrium $\tilde{\mathbf{s}}^{j}$ under which $\mathcal{A}_{j}$ assigns the task to the slow machine (recall that the task cannot be assigned to a dummy machine). Then, since $\mathbf{t}^{j}$ is a permutation of $\tilde{\mathbf{t}}^{j}$ and canonical vectors do not have ties, due to anonymity (see Definition 2) there has to exist an equilibrium $\hat{\mathbf{s}}^{j}$ with respect to $\mathbf{t}^{j}$, under which the task is given to the slow machine; this is a contradiction.

Claim 5. Mechanism $\mathcal{M}$ has at least $n-1$ well-behaved tasks. 
Proof. Let $\mathbf{t}$ be a true instance whose task cost vectors are all canonical. For example, let

$$
\mathbf{t}=\underbrace{\left(\begin{array}{cccc}
1 & 1 & \cdots & 1 \\
\alpha & \alpha & \cdots & \alpha \\
\infty & \infty & \cdots & \infty \\
\vdots & \vdots & & \vdots \\
\infty & \infty & \cdots & \infty
\end{array}\right)}_{2 n-3}
$$

Due to Claim 4, it is enough to show that there exists a fixed set of (at least) $n-1$ tasks which, under all equilibria of $\mathbf{t}$, they all get allocated to their fast machines. Formally, to get to a contradiction, assume that for any subset of tasks $\bar{J} \subseteq[m]$ with $|\bar{J}|=n-1$, there exists a task $j=\mathfrak{j}(\bar{J}) \in \bar{J}$ and an equilibrium $\mathbf{s}^{j}$ of $\mathbf{t}^{j}$ on which $\mathcal{A}_{j}$ allocates task $j$ to the slow machine. Then, since instance $\mathbf{t}$ has at least $m \geq 2 n-3$ tasks, we can apply this property repeatedly in order to get a sequence of $n-1$ tasks

$$
j_{1}=\mathfrak{j}([n-1]), \quad j_{2}=\mathfrak{j}\left([n] \backslash j_{1}\right), \quad \ldots, \quad j_{n-1}=\mathfrak{j}\left([2 n-3] \backslash\left\{j_{1}, j_{2}, \ldots, j_{n-2}\right\}\right)
$$

and corresponding equilibria $\mathbf{s}^{j_{1}}, \mathbf{s}^{j_{2}}, \ldots, \mathbf{s}^{j_{n-1}}$ (with respect to true canonical cost vectors $\mathbf{t}^{j_{1}}, \mathbf{t}^{j_{2}}, \ldots, \mathbf{t}^{j_{n-1}}$, respectively) with the property that, for all $\ell=1, \ldots, n-1$, mechanism $\mathcal{A}_{j_{\ell}}$ allocates task $j_{\ell}$ to the slow machine.

Without loss, assuming for the rest of this claim's proof that $\left\{j_{1}, j_{2}, \ldots, j_{n-1}\right\}=[n-1]$, we consider the following new profile of true costs

$$
\tilde{\mathbf{t}}=\left(\begin{array}{ccccc|c}
1 & \infty & \infty & \cdots & \infty \\
\infty & 1 & \infty & \cdots & \infty \\
\vdots & \ddots & \ddots & \ddots & \vdots & 0 \\
\infty & \cdots & \infty & 1 & \infty & \\
\alpha & \alpha & \cdots & \alpha & 1 &
\end{array}\right)
$$

where tasks $j=1, \ldots, n-1$ have canonical cost vectors with the fast machine at the diagonal and the slow one always being $i=n$; task $j=n$ is arbitrarily slow on all machines except $i=n$, for which it has a cost of 1 ; and all remaining tasks $j \geq n+1$ have been rendered essentially irrelevant by setting their running times to 0 for all machines.

Since all canonical vectors $\tilde{\mathbf{t}}^{j}, j=1, \ldots, n-1$, of the new true profile $\tilde{\mathbf{t}}$ are permutations of the ones in the original true profile $\mathbf{t}$, and additionally we established that there exist equilibria $\mathbf{s}^{j}$ with respect to $\mathbf{t}$ that assign all these tasks to their slow machines, then due to anonymity (see Definition 2) there must also exist an equilibrium $\tilde{\mathbf{s}}$ of mechanism $\mathcal{M}$ (with respect to $\tilde{\mathbf{t}}$ ) on which all tasks $j=1, \ldots, n-1$ are given to the machine with cost $\alpha$. Furthermore, clearly task $j=n$ needs to be allocated to machine $i=n$ as well on $\tilde{\mathbf{s}}$, since she is the only one with bounded running time (recall that $\mathcal{M}$ has a bounded PoA).

Summarizing, equilibrium $\tilde{\mathbf{s}}$ assigns all tasks to the last machine, for a makespan of $(n-1)$. $\alpha+1$. On the other hand, the diagonal allocation on $\tilde{\mathbf{t}}$, i.e. giving each task to the machine with cost 1 would have given a makespan of 1 . This results in a $\operatorname{PoA}$ bound of at least $\operatorname{PoA}(\mathcal{M}) \geq$ $(n-1) \alpha+1$, which contradicts our initial assumptions about mechanism $\mathcal{M}$.

In light of Claim 5, without loss let's assume from now on that the first $n-1$ tasks are well-behaved, and consider the following profile of true costs

$$
\hat{\mathbf{t}}=\left(\begin{array}{ccccc|c}
\alpha & \infty & \infty & \cdots & \infty \\
\infty & \alpha & \infty & \cdots & \infty \\
\vdots & \ddots & \ddots & \ddots & \vdots & 0 \\
\infty & \cdots & \infty & \alpha & \infty & \\
1 & 1 & \cdots & 1 & \alpha &
\end{array}\right)
$$


which, in a similar way to that in the proof of Claim 5 for profile $\tilde{\mathbf{t}}$, we derived by permuting accordingly the canonical vectors of the first $n-1$ tasks of $\mathbf{t}$, setting the cost of task $j=n$ to be bounded only for the last machine and, finally, rendering all tasks $j>n$ irrelevant.

Fix now any equilibrium $\hat{\mathbf{s}}=\left(\hat{\mathbf{s}}_{1}, \ldots, \hat{\mathbf{s}}_{n-1}, \hat{\mathbf{s}}_{n}\right)$ of $\mathcal{M}$ under $\hat{\mathbf{t}}$. Since tasks $j=1, \ldots, n-1$ are well-behaved (see Definition 5), under $\hat{\mathbf{s}}$ mechanism $\mathcal{M}$ has to assign them all to the machine with running time 1 . Task $j=n$ needs to be allocated to machine $i=n$ as well (since $\operatorname{PoA}(\mathcal{M})$ is bounded). Thus, equilibrium $\hat{\mathbf{s}}$ results in a makespan of $(n-1) \cdot 1+\alpha$, while the diagonal allocation on $\hat{\mathbf{t}}$ has a makespan of $\alpha$. This results in a bound of $\operatorname{PoS}(\mathcal{M}) \geq \frac{n-1}{\alpha}+1$, concluding the proof of the theorem.

\subsection{Optimal Mechanisms on the Pareto Frontier}

Next, we will design a class of mechanisms, parameterized by a quantity $\alpha$ that will populate, in a smooth way, the boundary given by Theorem 6 . Thus, these mechanisms achieve trade-offs that lie on the Pareto frontier of inefficiency for the class of task-independent and anonymous mechanisms.

Definition 6 (Second-Price mechanism with $\alpha$-relative reserve price $\left(\mathcal{S P} \mathcal{P}_{\alpha}\right)$ ). For $\alpha \geq 1, \mathcal{S P} \mathcal{P}_{\alpha}$ is the task-independent mechanism that, for each task $j$ : finds a machine $k \in \arg \min _{i \in N} s_{i, j}$ and sets a reserve price at $r=\alpha \cdot s_{k, j}$; assigns the task to the fastest machine $\iota(j) \in \operatorname{argmin}_{i \in N} s_{i, j}$ (breaking ties-arbitrarily); pays machine $\iota(j)$ the amount $\min \left\{\min _{i \in N \backslash\{\iota(j)\}} s_{i, j}, r\right\}$; pays nothing to the remaining machines $N \backslash \iota(j)$.

Informally, for each task $j$, the mechanism sets a reserve price which is $\alpha$ times larger than the smallest declared processing time, allocates the task to the fastest machine (according to the declarations) and pays the machine the minimum of the second-smallest declared processing time and the reserve price. What this mechanism achieves in terms of the equilibria that it induces is the following: assume that we create a bucket of tasks with true processing times at most $\alpha$ times larger than the smallest true processing time. Then, in every equilibrium of the mechanism, task $j$ is allocated to some machine in the bucket and moreover, for any machine in the bucket, there exists some equilibrium under which $\mathcal{S P}_{\alpha}$ allocates the task to that machine. This is captured formally by the following two lemmas. Referencing our discussion in Section 3.1.1, we remark that in the case of $\mathrm{FP}=\mathcal{S P}_{1}$, the tasks can only be assigned to the fastest machine(s), and in the case of $\mathrm{SP}=\mathcal{S P}{ }_{\infty}$, the bucket contains the whole set of machines.

Lemma 2 ("Nothing outside the bucket"). In any equilibrium of $\mathcal{S P}_{\alpha}$, any task $j$ can only be assigned to a machine with processing time at most $\alpha \cdot \min _{i \in N} t_{i, j}$.

Proof. Since $\mathcal{S P}_{\alpha}$ is task-independent, it suffices to consider the equilibria of a single component $\mathcal{S P}_{\alpha}^{j}$, corresponding to task $j$. Let $\mathbf{s}^{j}$ be such an equilibrium and without loss of generality, assume that $t_{1, j} \in \arg \min _{i \in N} t_{i, j}$, i.e., machine 1 is the fastest machine according to the true processing times. Assume by contradiction that in $\mathbf{s}^{j}$, some task $\ell$ with real processing time $t_{\ell, j}>\alpha \cdot t_{1, j}$ is allocated task $j$ and let $s_{\ell, j}$ be its report. Since machine $\ell$ receives the task, it obviously holds that $s_{\ell, j} \in \arg \min _{i \in N} s_{i, j}$. We will consider two cases.

Case 1: $s_{\ell, j} \leq t_{1, j}$. In this case, the reserve price is set at $r=\alpha \cdot s_{\ell, j} \leq \alpha \cdot t_{1, j}$ and machine $\ell$ receives a payment of at most $\alpha \cdot t_{1, j}$. By assumption however, its true processing time is larger than $\alpha \cdot t_{i, j}$ and therefore the machine has negative utility. By deviating to telling the truth, the machine can obtain a nonnegative utility, contradicting the fact that $\mathbf{s}^{j}$ is an equilibrium.

Case 2: $s_{\ell, j}>t_{1, j}$. In this case, machine 1 has 0 utility, since she is not allocated the task and she is not paid anything. However, if machine 1 deviates to telling the truth, (i.e., if she deviates to $s_{i, j}^{\prime}=t_{1, j}$ ), then, since $s_{\ell, j}=\min _{i \in N} s_{i, j}$ by assumption, the machine will now win the task (i.e., $x_{1 j}=1$ ) and will receive a payment of $s_{\ell, j}>t_{1, j}$, obtaining strictly positive utility. Again, this contradicts the fact that $\mathbf{s}^{j}$ is an equilibrium. 
In any case, $\mathbf{s}^{j}$ can not be an equilibrium in which task $j$ is allocated to some machine with processing time larger than $\alpha \cdot \min _{i \in N} t_{i, j}$.

Lemma 3 ("Everything inside the bucket"). For every input profile $\mathbf{t}$ and any $\alpha>1$, there exist equilibria of $\mathcal{S P}_{\alpha}$ such that for every task $j$, every machine with processing time at most $\alpha \cdot \min _{i \in N} t_{i, j}$ can be allocated task $j$.

Proof. Again, since $\mathcal{S P}_{\alpha}$ is task-independent, it suffices to consider the equilibria of a single component $\mathcal{S P}_{\alpha}^{j}$, corresponding to task $j$. Given an input profile $\mathbf{t}$, let $J_{\alpha}$ be the set of machines $i^{\prime}$ such that $t_{i^{\prime}, j} \leq \alpha \cdot \min _{i \in N} t_{i, j}$ and let $t_{f}=\min _{i \in N} t_{i, j}$ be the processing time of the fastest machine for task $j$. Let $k$ be any machine in $J_{\alpha}$; we will consider two strategy profiles (or rather their restrictions to the $j$-th component), depending on whether $t_{k, j}>t_{f}$ or $t_{k, j}=t_{f}$.

Case 1: $t_{k, j}>t_{f}$. Then, consider the strategy profile $\mathbf{s}^{j}$ such that $s_{k, j}=t_{f}$ and $s_{i, j}=t_{k, j}$ for any machine $i \in J \backslash\{k\}$. We will prove that $\mathbf{s}^{j}$ is an equilibrium, by considering possible deviations of machine $k$, and the remaining machines in $J \backslash\{k\}$ separately.

a) The current utility of machine $k$ is 0 , since it receives the task for which the true processing time is $t_{k, j}$ and receives a payment of $t_{k, j}$. Note that the reserve price is set to at most $t_{k, j}$, since $t_{k, j} \leq \alpha \cdot t_{f}$ by assumption, and $s_{k, j}=t_{f}$. Since there exist machines with reported processing times at $t_{k, j}$, machine $k$ can not obtain positive utility by any deviation, even if it increases the reserve price while still winning the task.

b) Consider any machine $i \in J \backslash\{k\}$. Since machine $i$ is not winning the task, its utility is 0 , and the only way to possibly obtain a positive utility is by forcing an allocation in which it wins the task. For this to be possible, it has to deviate to $s_{i, j}^{\prime} \leq t_{f}=s_{k, j}$, as otherwise machine $k$ would still be the winner. In that case however, the payment of machine $i$ will be at most $t_{f}$ and by assumption, we know that $t_{i, j} \geq t_{f}$ for any task $j \in J$. Therefore, the deviation results in a utility of at most 0 for machine $i$ and it is not a beneficial deviation.

Case 2: $t_{k, j}=t_{f}$. Then, consider the strategy profile $\mathbf{s}^{j}$ such that $s_{k, j}=t_{f}$ and $s_{i, j}=t_{f}+\varepsilon$, where $t_{f}+\varepsilon<\alpha \cdot t_{f}$; this is possible since $\alpha>1$ and the continuity of the strategy space. Again, we consider possible deviations of machine $k$, and the remaining machines separately.

a) The current utility of machine $k$ is $\varepsilon$, since it receives the task for which the true processing time is $t_{k, j}=t_{f}$ and receives a payment of $t_{f}+\varepsilon$, the second smallest reported processing time (which is also smaller than the reserve price by the choice of $\varepsilon$ ). In order for machine $k$ to still receive the task, it has to use some strategy $s_{k, j}^{\prime} \leq t_{f}+\varepsilon$, but any such strategy can not affect the payment that it receives. Therefore the machine does not have a beneficial deviation.

b) The argument in this case is identical to Case $1 \mathrm{~b}$ above.

Theorem 7. The Price of Anarchy of $\mathcal{S P}_{\alpha}$ on $n$ machines is at most $(n-1) \alpha+1$.

Proof. Fix some underlying $n \times m$ true cost matrix $\mathbf{t}$ and a parameter $\alpha>1$. Fix also an optimal (makespan-minimizing) allocation OPT of $\mathbf{t}$ and a (pure) Nash equilibrium $\mathbf{s}$ of $\mathcal{S P}_{\alpha}$ under true costs t. For any task $j=1, \ldots, m$, let $\iota^{*}(j)$ and $\iota(j)$ denote the machine that gets task $j$ at OPT and $\mathcal{S P} \mathcal{P}_{\alpha}(\mathbf{s})$, respectively. Also, let $K_{i}^{*}, K_{i}$ denote the corresponding machine loads and $J_{i}^{*}, J_{i}$ the sets of assigned tasks; that is, for $i=1, \ldots, n$, we define

$$
K_{i}^{*} \equiv \sum_{j \in J_{i}^{*}} t_{i, j} \quad \text { and } \quad K_{i} \equiv \sum_{j \in J_{i}} t_{i, j}
$$


where

$$
J_{i}^{*}=\left\{j \in J \mid \iota^{*}(j)=i\right\} \quad \text { and } \quad J_{i}=\{j \in J \mid \iota(j)=i\} .
$$

Finally, it is without loss of generality to assume that $K_{1} \geq K_{2} \geq \cdots \geq K_{n}$, so that the makespan of $\mathcal{S P}_{\alpha}$ on $\mathbf{s}$ is

$$
K_{1}=\sum_{j \in J_{1}} t_{1, j}=\sum_{j \in J_{1} \cap J_{1}^{*}} t_{1, j}+\sum_{j \in J_{1} \backslash J_{1}^{*}} t_{1, j} \leq \sum_{j \in J_{1}^{*}} t_{1, j}+\alpha \sum_{j \in J \backslash J_{1}^{*}} t_{\iota^{*}(j), j},
$$

the last inequality holding since, for any task $j, t_{\iota(j), j} \leq \alpha \cdot t_{\iota^{*}(j), j}$ (due to Lemma 2). Thus, we can bound our mechanism's makespan by

$$
K_{1} \leq K_{1}^{*}+\alpha \sum_{i=2}^{n} K_{i}^{*} \leq K_{1}^{*}+\alpha(n-1) \max _{i=2, \ldots, n} K_{i}^{*}
$$

Putting everything together, and denoting for simplicity $x=K_{1}^{*}$ and $y=\max _{i=2, \ldots, n} K_{i}^{*}$, the PoA of $\mathcal{S P} \mathcal{P}_{\alpha}$ is finally upper bounded by

$$
\operatorname{PoA}\left(\mathcal{S P}_{\alpha}\right) \leq \frac{x+\alpha(n-1) y}{\max _{i=1, \ldots, n} K_{i}^{*}}=\frac{x+\alpha(n-1) y}{\max \{x, y\}} \leq \alpha(n-1)+1,
$$

the last step coming from applying Lemma 4 (see appendix) with $\beta=\alpha(n-1)$ and $\gamma=1$.

Theorem 8. The Price of Stability of $\mathcal{S P}_{\alpha}$ on $n$ machines is at most $\frac{n-1}{\alpha}+1$.

Proof. Fix some underlying $n \times m$ true cost matrix $\mathbf{t}$, an optimal (makespan-minimizing) allocation OPT of $\mathbf{t}$ and a parameter $\alpha>1$. Also, let $\iota^{*}(j)$ denote the machine that gets task $j$ at OPT.

We partition the set of tasks $J=\{1,2, \ldots, m\}$ into two sets $J_{\text {small }}$ and $J_{\text {large }}$, based on their processing time under OPT. More specifically, we define:

$J_{\text {small }} \equiv\left\{j \in J \mid t_{\iota^{*}(j), j} \leq \alpha \cdot \min _{i \in N} t_{i, j}\right\} \quad$ and $\quad J_{\text {large }} \equiv J \backslash J_{\text {small }}=\left\{j \in J \mid t_{\iota^{*}(j), j}>\alpha \cdot \min _{i \in N} t_{i, j}\right\}$.

Intuitively, in light of Lemmas 2 and 3, we can think of $J_{\text {small }}$ as containing all the tasks that $\mathcal{S P} \mathcal{P}_{\alpha}$ can allocate to the same machine as OPT in some equilibrium and $J_{\text {large }}$ containing the tasks for which this is not possible, but which nevertheless end up at a machine that runs them faster than in OPT.

Now consider the pure Nash equilibrium $\mathbf{s}$ of $\mathcal{S P}_{\alpha}$ (with respect to true running times $\mathbf{t}$ ) that allocates all small tasks at the same machine as OPT, and all large tasks myopically to the fastest machine for that task. More precisely, if $\iota(j)$ denotes the machine that gets task $j$ under $\mathcal{S P}_{\alpha}(\mathbf{s})$, we set $\iota(j)=\iota^{*}(j)$ for all $j \in J_{\text {small }}$ and $\iota(j) \in \operatorname{argmin}_{i \in N} t_{i, j}$ for $j \in J_{\text {large }}$. The fact that such an equilibrium indeed exists, is a consequence of Lemma 3.

Let $K_{i}$ denote the load of machine $i$ after allocating only the small tasks according to OPT (and thus, also according to the equilibrium $\mathbf{s}$ of $\mathcal{S P} \mathcal{P}_{\alpha}$ ). Let $L_{i}^{*}, L_{i}$ denote the load of machine $i$ after allocating only the large tasks according to OPT and $\mathbf{s}$, respectively. That is, we formally define:

$$
K_{i} \equiv \sum_{j \in J_{\text {small }}: \iota(j)=i} t_{i, j}, \quad L_{i}^{*} \equiv \sum_{j \in J_{\text {large }}: \iota^{*}(j)=i} t_{i, j} \quad \text { and } \quad L_{i} \equiv \sum_{j \in J_{\text {large }: \iota(j)=i}} t_{i, j} .
$$

Without loss, let's assume that $K_{1} \geq K_{2} \geq \cdots \geq K_{n}$. Then, the makespan of OPT is

$$
\max _{i \in N}\left(K_{i}+L_{i}^{*}\right) \geq \max \left\{K_{1}+L_{1}^{*}{ }_{i=2, \ldots, n} L_{i}^{*}\right\} \geq \max \left\{K_{1}+L_{1}^{*}, \frac{1}{n-1} \sum_{i=2, \ldots, n} L_{i}^{*}\right\},
$$


while that of $\mathbf{s}$ can be upper bounded by

$$
\max _{i \in N}\left(K_{i}+L_{i}\right) \leq \max _{i \in N} K_{i}+\max _{i \in N} L_{i} \leq K_{1}+\sum_{i=1}^{n} L_{i} \leq K_{1}+\frac{1}{\alpha} \sum_{i=1}^{n} L_{i}^{*} \leq K_{1}+L_{1}^{*}+\frac{1}{\alpha} \sum_{i=2}^{n} L_{i}^{*},
$$

where the second to last inequality holds due to the fact that for large tasks

$$
\sum_{i=1}^{n} L_{i}=\sum_{j \in J_{\text {large }}} t_{\iota(j), j}=\sum_{j \in J_{\text {large }}} \min _{i \in N} t_{i, j} \leq \sum_{j \in J_{\text {large }}} \frac{1}{\alpha} t_{\iota^{*}(j), j}=\frac{1}{\alpha} \sum_{i=1}^{n} L_{i}^{*},
$$

and the last one holds due to $\alpha \geq 1$.

Putting everything together, and denoting for simplicity $x=K_{1}+L_{1}^{*}$ and $y=\sum_{i=2}^{n} L_{i}^{*}$, we have that

$$
\operatorname{PoS}\left(\mathcal{S P}_{\alpha}\right) \leq \frac{x+\frac{1}{\alpha} y}{\max \left\{x, \frac{1}{n-1} y\right\}} \leq \frac{n-1}{\alpha}+1,
$$

the last inequality holding by applying Lemma 4 with $\beta=\frac{1}{\alpha}$ and $\gamma=\frac{1}{n-1}$.

\section{$5 \quad$ Discussion and Future Directions}

In this section, we discuss some implications of our approach, as well as directions for future work. On a general level, one could follow our agenda of studying the inefficiency trade-off between the Price of Anarchy and the Price of Stability for many other problems in algorithmic mechanism design, such as auctions [38, 54], machine scheduling without money [23, 31], or resource allocation [11], to name a few, for which the two inefficiency notions have already been studied separately.

In terms of the strategic scheduling setting, our work gives rise to a plethora of intriguing questions for future work, both on a technical and a conceptual level, which we highlight below in more detail.

\subsection{General Mechanisms}

Why did we focus on task-independent mechanisms for our tight frontier result, since they are seemingly not a good fit for makespan minimization? First of all, the latter statement is true for the algorithmic version of the problem, but not necessarily true for the strategic version. It is not only conceivable that task-independent mechanisms, despite their "naive" allocation rules, can induce equilibria in which the allocation is actually quite efficient, but this actually happens, as evidenced by the Price of Stability of the Second Price Mechanism. Also, task-independent mechanisms are much more amenable to an equilibrium analysis, because each task induces a separate games between the machines. Showing the limitations of this class is quite important, because it is not a priori clear that they could not achieve the best possible trade-offs suggested by Theorem 2 .

The major open question associated with our work is whether there exists a mechanism that achieves a better trade-off than that of Theorem 6 , or in other words,

\section{"Is the yellow region of Fig. 3 empty or not?"}

If such a mechanism exists, it will most probably not be task-independent ${ }^{11}$; this is somewhat reminiscent of the state-of-the-art results in truthful machine scheduling, where the best possible mechanisms (with respect to the approximation ratio, see Section 2.2) for several variants of the problem are in fact task-independent $[10,45]$ and whether a better mechanism that is not task-independent exists is a prominent open question.

\footnotetext{
${ }^{11}$ See also the discussion in Section 5.2.
} 
While in the case of truthful mechanisms, the general consensus ${ }^{12}$ seems to be that the best achievable mechanisms will in fact eventually proven to be task-independent, the situation in the strategic version of the problem might be quite different. This is because we have all possible mechanisms at our disposal and it is more conceivable that some allocation rule, tied with some appropriate payment function could potentially outperform the trade-off bounds of Theorem 6. This seems, however, like a quite challenging task; to offer some intuition, we remark the following about the design of general mechanisms for the problem.

The most natural idea is perhaps to use a known algorithm for unrelated machine scheduling $[14,28,36]$ such as the greedy allocation algorithm ${ }^{13}$ of Ibarra and Kim [28], or even the makespan-optimal algorithm and couple them with a "get-paid-your-load" payment function, where each machine receives a monetary compensation equal to the sum of the reported processing times for the tasks that she gets assigned. This is essentially the generalization of the payment rule of the First-Price mechanism for more general allocation rules. The hope is that by virtue of having a more efficient allocation rule, the resulting mechanism will always have equilibria with small makespan (good PoS) while never having equilibria with very large makespan (good PoA). Unfortunately, one can show that for a large class of such allocation algorithms (which includes all the aforementioned algorithms that have been proposed in the literature for the classical unrelated machine scheduling problem), the Price of Anarchy of the resulting mechanism will be unbounded.

Overall, for a mechanism to lie in the yellow region of Fig. 3, it seems imperative that it will need to employ some more complicated payment function, which will "guide" the agents towards the desired equilibria, rather than simply attempt to implement a better allocation rule with known payment structures.

\subsection{The Role of Anonymity}

In the proof of Theorem 6 , we used the fact that the mechanism in question is anonymous. In many cases in the general related literature, this assumption is without loss of generality, as the best possible mechanisms with respect to an objective are anonymous. In the literature of the unrelated machine scheduling problem in algorithmic mechanism design however, understanding the role of anonymity is a long-standing open problem. In particular, while all the known mechanisms for several variants of the problem are anonymous, the best-known lower bounds for anonymous and non-anonymous mechanisms are strikingly different, from, specifically $n$ in the former case (given by [3] and matching the best-known upper bound of [45]) and 2.75 in the latter case (given by [24]). These challenges are inherited in the strategic setting as well. Nevertheless, for general (not necessarily anonymous) task-independent mechanisms, we can still show the following inefficiency trade-off. Its proof can be found in Appendix B.

Theorem 9. For any task-independent scheduling mechanism $\mathcal{M}$ for $n$ machines, and real $\alpha>1$,

$$
\operatorname{PoA}(\mathcal{M})<(n-1) \frac{\alpha}{\sqrt{2}}+1 \quad \Longrightarrow \quad \operatorname{PoS}(\mathcal{M}) \geq \frac{(n-1)}{\alpha \sqrt{2}}+1
$$

The above is a strict improvement with respect to the general boundary given by Theorem 2 , but still does not quite match the Pareto frontier that we proved for anonymous mechanisms. In other words, the inefficiency boundary given by Theorem 9 lies strictly within the yellow area in Fig. 3. Obtaining tight bounds is an interesting open problem.

\footnotetext{
${ }^{12}$ This is because of the result of Ashlagi et al. [3] for anonymous mechanisms and due to personal communications with authors of central papers in the field.

${ }^{13}$ The algorithm is referred to as "Algorithm D" in [28].
} 


\subsection{Equilibrium Notion Considerations}

In this paper, we study the set of all possible equilibria of mechanisms for the problem, which may include equilibria which are weakly dominated (e.g. see [42, Sec. 1.8]), i.e., the agents could use a different strategy instead of their equilibrium strategy and obtain the same utilities, regardless of the reports of the other agents. These type of equilibria are known to exist in the Second-Price mechanism and our class of mechanisms $\mathcal{S P}_{\alpha}$ also exhibits such equilibria. In order to quantify these type of equilibria in terms of the agents' aversion to risk, Babaioff et al. [4] defined the notion of exposure factor, which measures the amount of risk that an agent is willing to expose herself to, when best-responding. In the terminology of our setting, the exposure $\gamma$ of strategy $s_{i}$ is such that

$$
p_{i}\left(s_{i}, s_{-i}\right) \geq(1+\gamma) \sum_{i \in S_{i}} t_{i}
$$

where $s_{-i}$ is any vector of strategies of the other machines and $S_{i}$ is the set of tasks assigned to machine $i$ under $\mathbf{x}\left(s_{i}, s_{-i}\right)$. In simple words, $\gamma$ is used to quantify how much extra cost agent $i$ could possibly experience, if all other agents coordinated to a strategy that is the worst possible for the agent. Then, Babaioff et al. [4] proceed to define the set $\mathcal{Q}_{t}^{\gamma}$ as the set of all equilibria that consist only of strategies with exposure factor at most $\gamma$ and the corresponding notion of the Price of Anarchy with the respect to this equilibrium set. Given a parameter $\alpha$, mechanism $\mathcal{S P} \mathcal{P}_{\alpha}$ can be seen as achieving a PoS guarantee even with respect to the set $Q_{t}^{\alpha-1}$ of equilibria consisting of strategies of exposure at most $\alpha-1$. Conceptually, even if one is only willing to accept a certain level of risk exposure, an appropriate $\beta$ can be chosen and the corresponding mechanisms $\mathcal{S P} \mathcal{P}_{\alpha}$ for $\alpha \leq \beta$, will lie on the inefficiency boundary, even if the solution concept is the $\gamma$-exposure Nash equilibrium.

Whether there exists a mechanism that can match, in undominated Nash equilibria, the guarantees of $\mathcal{S} \mathcal{P}_{\alpha}$, is an interesting open question; we believe though that this is rather unlikely. That being said, proving impossibility results for general classes of mechanisms seems quite challenging, as the property of not being dominated does not convey much information from a technical standpoint and in particular has different implications for different mechanisms. This is in contrast with the more standard approach in auctions, where specific mechanisms have been studied, for which undominated strategies imply a very handy non-overbidding property, e.g., see $[17,39]$.

\subsection{Computational Considerations}

As we mentioned earlier, the mechanisms that we construct in this paper (see Definition 6) are rather simple and run in polynomial time, and this is actually the case for all known mechanisms for the truthful scheduling problem as well. It would be interesting to investigate whether adding computational efficiency as a desirable property of the mechanisms in question can have any implications on the inefficiency boundary. For truthful scheduling, this is unlikely to be an issue, since the constraint of truthfulness itself typically leads to rather simple mechanisms which are easily seen to be efficient. As we hinted in Section 5.1 however, it is conceivable that from the space of all possible mechanisms that we can use, the best one might employ an allocation algorithm that is computationally intractable. Concretely, it could be possible that the makespan-optimal algorithm (which does not run in polynomial time, unless $\mathrm{P}=\mathrm{NP}[36]$ ) can be coupled with an appropriate payment function to achieve a better trade-off guarantee. From this discussion, we deduce the following, very interesting question:

\footnotetext{
"If we add computational efficiency as a constraint, can we prove a stronger inefficiency boundary than that of Fig. 2?."
} 
Conceptually, the question above regards whether there is a fundamental connection between the running time of the allocation rule and the PoA/PoS trade-off that can be explored via a corresponding inefficiency boundary.

Acknowledgements We are grateful to the anonymous reviewers of the journal version of this paper for their careful reading of our manuscript and for their valuable comments that helped us to simplify and improve the presentation at key points of our work. We also thank Elias Koutsoupias, Maria Kyropoulou and Diogo Poças for useful discussions.

\section{References}

[1] E. Anshelevich, A. Dasgupta, J. Kleinberg, É. Tardos, T. Wexler, and T. Roughgarden. The price of stability for network design with fair cost allocation. SIAM Journal on Computing, 38(4):1602-1623, 2008. doi:10.1137/07068009.

[2] A. Archer and É. Tardos. Truthful mechanisms for one-parameter agents. In Proceedings of the 42nd IEEE symposium on Foundations of Computer Science (FOCS), pages 482-491, 2001. doi:10.1109/sfcs.2001.959924.

[3] I. Ashlagi, S. Dobzinski, and R. Lavi. Optimal lower bounds for anonymous scheduling mechanisms. Mathematics of Operations Research, 37(2):244-258, 2012. doi:10.1287/moor.1110.0534.

[4] M. Babaioff, B. Lucier, N. Nisan, and R. Paes Leme. On the efficiency of the Walrasian mechanism. In Proceedings of the 15th ACM Conference on Economics and Computation (EC), pages 783-800, 2014. doi:10.1145/2600057.2602850.

[5] I. Caragiannis, C. Kaklamanis, P. Kanellopoulos, M. Kyropoulou, B. Lucier, R. P. Leme, and É. Tardos. Bounding the inefficiency of outcomes in generalized second price auctions. Journal of Economic Theory, 156:343-388, 2015. doi:10.1016/j.jet.2014.04.010.

[6] S. Chawla, J. D. Hartline, D. Malec, and B. Sivan. Prior-independent mechanisms for scheduling. In Proceedings of the 45th Annual ACM Symposium on Theory of Computing (STOC), pages 51-60, 2013. doi:10.1145/2488608.2488616.

[7] G. Christodoulou and E. Koutsoupias. Mechanism design for scheduling. Bulletin of the EATCS, 97:40-59, 2009.

[8] G. Christodoulou, E. Koutsoupias, and A. Vidali. A characterization of 2-player mechanisms for scheduling. In 16th Annual European Symposium on Algorithms (ESA), pages 297-307, 2008. doi:10.1007/978-3-540-87744-8.

[9] G. Christodoulou, E. Koutsoupias, and A. Vidali. A lower bound for scheduling mechanisms. Algorithmica, 55(4):729-740, 2009. doi:10.1007/s00453-008-9165-3.

[10] G. Christodoulou, E. Koutsoupias, and A. Kovács. Mechanism design for fractional scheduling on unrelated machines. ACM Transactions on Algorithms, 6(2):38:1-38:18, 2010. doi: $10.1145 / 1721837.1721854$.

[11] G. Christodoulou, A. Filos-Ratsikas, S. K. S. Frederiksen, P. W. Goldberg, J. Zhang, and J. Zhang. Social welfare in one-sided matching mechanisms (extended abstract). In Proceedings of the International Conference on Autonomous Agents and Multiagent Systems (AAMAS), pages 1297-1298, 2016. doi:10.1007/978-3-319-46882-2_3. 
[12] E. H. Clarke. Multipart pricing of public goods. Public Choice, 11(1):17-33, 1971. doi:10.1007/bf01726210.

[13] C. Daskalakis and S. M. Weinberg. Bayesian truthful mechanisms for job scheduling from bi-criterion approximation algorithms. In Proceedings of the 26th annual ACM-SIAM Symposium on Discrete Algorithms (SODA), pages 1934-1952, 2015. doi:10.1137/1.9781611973730.130.

[14] E. Davis and J. M. Jaffe. Algorithms for scheduling tasks on unrelated processors. Journal of the ACM, 28(4):721-736, 1981. doi:10.1145/322276.322284.

[15] S. Dobzinski and A. Shaulker. Improved lower bounds for truthful scheduling. ArXiv, abs/2007.04362, 2020. URL https://arxiv.org/abs/2007.04362.

[16] S. Dobzinski and M. Sundararajan. On characterizations of truthful mechanisms for combinatorial auctions and scheduling. In Proceedings of the 9th ACM Conference on Electronic Commerce (EC), pages 38-47, 2008. doi:10.1145/1386790.1386798.

[17] M. Feldman, B. Lucier, and N. Nisan. Correlated and coarse equilibria of single-item auctions. In Proceedings of the 12th International Conference on Web and Internet Economics (WINE), pages 131-144, 2016. doi:10.1007/978-3-662-54110-4_10.

[18] A. Filos-Ratsikas, S. K. S. Frederiksen, and J. Zhang. Social welfare in one-sided matchings: Random priority and beyond. In Proceedings of the 7th International Symposium on Algorithmic Game Theory (SAGT), pages 1-12, 2014. doi:10.1007/978-3-662-44803-8_1.

[19] A. Filos-Ratsikas, Y. Giannakopoulos, and P. Lazos. The Pareto frontier of inefficiency in mechanism design. In Proceedings of the 15th Conference on Web and Internet Economics (WINE), pages 186-199, 2019. doi:10.1007/978-3-030-35389-6_14.

[20] M. Gairing. Covering games: Approximation through non-cooperation. In Proceedings of the 5th International Workshop on Internet and Network Economics (WINE), pages 184-195, 2009. doi:10.1007/978-3-642-10841-9_18.

[21] M. R. Garey, D. S. Johnson, and R. Sethi. The complexity of flowshop and jobshop scheduling. Mathematics of Operations Research, 1(2):117-129, 1976 . URL http://www.jstor.org/stable/3689278.

[22] Y. Giannakopoulos and M. Kyropoulou. The VCG mechanism for bayesian scheduling. ACM Trans. Econ. Comput., 5(4):19:1-19:16, 2017. doi:10.1145/3105968.

[23] Y. Giannakopoulos, E. Koutsoupias, and M. Kyropoulou. The anarchy of scheduling without money. Theoretical Computer Science, 778:19-32, 2019. doi:10.1016/j.tcs.2019.01.022.

[24] Y. Giannakopoulos, A. Hammerl, and D. Poças. A new lower bound for deterministic truthful scheduling. In Proceedings of the 13th Symposium on Algorithmic Game Theory (SAGT), 2020. doi:10.1007/978-3-030-57980-7_15.

[25] R. L. Graham. Bounds for certain multiprocessing anomalies. Bell System Technical Journal, 45(9):1563-1581, nov 1966. doi:10.1002/j.1538-7305.1966.tb01709.x.

[26] T. Groves. Incentives in teams. Econometrica, 41(4):617-631, 1973. doi:10.2307/1914085.

[27] L. A. Hall. Approximation algorithms for scheduling. In D. S. Hochbaum, editor, Approximation Algorithms for NP-hard Problems, pages 1-45. PWS Publishing Company, 1997. 
[28] O. H. Ibarra and C. E. Kim. Heuristic algorithms for scheduling independent tasks on nonidentical processors. Journal of the ACM, 24(2):280-289, 1977. doi:10.1145/322003.322011.

[29] J. R. Jackson. Scheduling a production line to minimize maximum tardiness. Research Report 43, Mgmt. Sci. Research Project, University of California, Los Angeles, 1955.

[30] S. M. Johnson. Optimal two-and three-stage production schedules with setup times included. Naval research logistics quarterly, 1(1):61-68, 1954. doi:10.1002/nav.3800010110.

[31] E. Koutsoupias. Scheduling without payments. Theory of Computing Systems, 54(3): 375-387, 2014. doi:10.1007/s00224-013-9473-0.

[32] E. Koutsoupias and C. Papadimitriou. Worst-case equilibria. Computer Science Review, 3 (2):65-69, 2009. doi:10.1016/j.cosrev.2009.04.003.

[33] E. Koutsoupias and A. Vidali. A lower bound of $1+\phi$ for truthful scheduling mechanisms. Algorithmica, 66(1):211-223, 2013. doi:10.1007/s00453-012-9634-6.

[34] R. Lavi and C. Swamy. Truthful mechanism design for multidimensional scheduling via cycle monotonicity. Games and Economic Behavior, 67(1):99-124, 2009. doi:10.1016/j.geb.2008.08.001.

[35] J. Lenstra, A. Rinnooy Kan, and P. Brucker. Complexity of machine scheduling problems. In P. Hammer, E. Johnson, B. Korte, and G. Nemhauser, editors, Studies in Integer Programming, volume 1 of Annals of Discrete Mathematics, pages 343-362. 1977. doi:10.1016/S0167-5060(08)70743-X.

[36] J. K. Lenstra, D. B. Shmoys, and É. Tardos. Approximation algorithms for scheduling unrelated parallel machines. Mathematical Programming, 46(1):259-271, 1990. doi:10.1007/bf01585745.

[37] P. Lu and C. Yu. Randomized truthful mechanisms for scheduling unrelated machines. In Proceedings of the 4 th International Workshop on Internet and Network Economics (WINE), pages 402-413, 2008. doi:10.1007/978-3-540-92185-1_46.

[38] B. Lucier, Y. Singer, V. Syrgkanis, and E. Tardos. Equilibrium in combinatorial public projects. In Proceedings of the 9th International Conference on Web and Internet Economics (WINE), pages 347-360, 2013. doi:10.1007/978-3-642-45046-4_28.

[39] E. Markakis and O. Telelis. Uniform price auctions: Equilibria and efficiency. Theor. Comp. Sys., 57(3):549--575, 2015. doi:10.1007/s00224-014-9537-9.

[40] A. Mu'alem and M. Schapira. Setting lower bounds on truthfulness. Games and Economic Behavior, 110:174-193, 2018. doi:10.1016/j.geb.2018.02.001.

[41] R. B. Myerson. Optimal auction design. Mathematics of Operations Research, 6(1):58-73, 1981. doi:10.1287/moor.6.1.58.

[42] R. B. Myerson. Game Theory: Analysis of Conflict. Harvard University Press, 1997.

[43] N. Nisan. Introduction to mechanism design (for computer scientists). In N. Nisan, T. Roughgarden, É. Tardos, and V. Vazirani, editors, Algorithmic Game Theory, chapter 9. Cambridge University Press, 2007.

[44] N. Nisan and A. Ronen. Algorithmic mechanism design. Games and Economic Behavior, 35(1/2):166-196, 2001. doi:10.1006/game.1999.0790. 
[45] N. Nisan and A. Ronen. Computationally feasible VCG mechanisms. Journal of Artificial Intelligence Research, 29(1):19-47, 2007. doi:10.1613/jair.2046.

[46] M. L. Pinedo. Scheduling. Springer, 2012. doi:10.1007/978-1-4614-2361-4.

[47] C. N. Potts and V. A. Strusevich. Fifty years of scheduling: a survey of milestones. Journal of the Operational Research Society, 60(1):S41-S68, 2009. doi:10.1057/jors.2009.2.

[48] V. Ramaswamy, D. Paccagnan, and J. R. Marden. Multiagent coverage problems: The trade-off between anarchy and stability. CoRR, abs/1710.01409, July 2018. URL http://arxiv.org/abs/1710.01409.

[49] A. H. G. Rinnooy Kan. Machine Scheduling Problems. Springer, 1976. doi:10.1007/978-1-4613-4383-7.

[50] T. Roughgarden and É. Tardos. How bad is selfish routing? J. ACM, 49(2):236-259, 2002. doi: $10.1145 / 506147.506153$.

[51] T. Roughgarden, V. Syrgkanis, and E. Tardos. The price of anarchy in auctions. Journal of Artificial Intelligence Research, 59:59-101, 2017. doi:10.1613/jair.5272.

[52] M. Saks and L. Yu. Weak monotonicity suffices for truthfulness on convex domains. In Proceedings of the 6th ACM Conference on Electronic Commerce (EC), pages 286-293, 2005. doi:10.1145/1064009.1064040.

[53] A. S. Schulz and N. S. Moses. On the performance of user equilibria in traffic networks. In Proceedings of the 14th Annual ACM-SIAM Symposium on Discrete Algorithms (SODA), pages 86-87, 2003.

[54] V. Syrgkanis and E. Tardos. Composable and efficient mechanisms. In Proceedings of the 45th Annual ACM Symposium on Theory of Computing (STOC), pages 211-220, 2013. doi:10.1145/2488608.2488635.

[55] W. Vickrey. Counterspeculation, auctions and competitive sealed tenders. Journal of Finance, 16(1):8-37, 1961. doi:10.1111/j.1540-6261.1961.tb02789.x.

\section{Appendix}

\section{A Technical Lemmas}

Lemma 4. For any nonnegative reals $x, y$ with $x y \neq 0$ and all positive reals $\beta, \gamma$ :

$$
\frac{x+\beta y}{\max \{x, \gamma y\}} \leq \frac{\beta}{\gamma}+1
$$

Proof. There are two cases to consider. First, if $x \geq \gamma y$, then

$$
\frac{x+\beta y}{\max \{x, \gamma y\}}=\frac{x+\beta y}{x}=\beta \frac{y}{x}+1 \leq \beta \frac{1}{\gamma}+1 .
$$

Secondly, if $\gamma y \geq x$, then

$$
\frac{x+\beta y}{\max \{x, \gamma y\}}=\frac{x+\beta y}{\gamma y}=\frac{\beta}{\gamma}+\frac{x}{\gamma y} \leq \frac{\beta}{\gamma}+1 .
$$


Lemma 5. For $i, j=1, \ldots, n$, let reals $\alpha>0, a_{i, j}>0($ for $i \neq j)$ and $a_{i, i}=0$ such that for all $j$ :

$$
\sum_{i=1}^{n} a_{i, j}<\frac{(n-1) \cdot \alpha}{\sqrt{2}} .
$$

Then, for any positive $\varepsilon \leq \frac{\alpha}{(n-1) \sqrt{2}}$ there exists some $i$ such that:

$$
\max _{\emptyset \neq I \subseteq[n] \backslash\{i\}} \frac{|I|}{\max _{j \in I} a_{i, j}+\varepsilon}>\frac{n-1}{\alpha \sqrt{2}} .
$$

The above inequality cannot be further improved.

Proof. We proceed using a proof by contradiction. For any fixed $i$, we use the index $i_{k}$ to refer to $a_{i, i_{k}}$, the $k$-th smallest among the $a_{i, j}$. By contradiction, we have that for all $i$ and nonempty $I \subseteq[n] \backslash\{i\}:$

$$
\frac{|I|}{\max _{j \in I} a_{i, j}+\varepsilon} \leq \frac{n-1}{\alpha \sqrt{2}}
$$

In particular, for all $I_{k}=\{2, \ldots, k\}$ (note that $a_{i, i_{1}}=a_{i, i}=0$ ) we get:

$$
\frac{k-1}{a_{i, i_{k}}+\varepsilon} \leq \frac{n-1}{\alpha \sqrt{2}} \Longrightarrow a_{i, i_{k}} \geq \alpha \frac{(k-1) \sqrt{2}}{n-1}-\varepsilon
$$

Summing over all values of $i$ and $j$ :

$$
\begin{aligned}
\sum_{i=1}^{n} \sum_{j=1}^{n} a_{i, j}=\sum_{i=1}^{n} \sum_{k=2}^{n} a_{i, i_{k}} & \geq \sum_{i=1}^{n} \sum_{k=2}^{n} \alpha \frac{(k-1) \sqrt{2}}{n-1}-\varepsilon \\
& =\frac{\alpha \sqrt{2}}{n-1} \sum_{i=1}^{n} \sum_{k=2}^{n} k-1-\varepsilon \\
& =\frac{\alpha \sqrt{2}}{n-1} \cdot n \cdot \frac{n(n-1)}{2}-n(n-1) \varepsilon \\
& =n \frac{n \cdot \alpha}{\sqrt{2}}-n(n-1) \varepsilon .
\end{aligned}
$$

Since the $a_{i j}$ are partitioned by the $n$ distinct values of $j$, there must be some $j$ for which:

$$
\sum_{i=1}^{n} a_{i, j} \geq \frac{n \cdot \alpha}{\sqrt{2}}-(n-1) \varepsilon \geq \frac{(n-1) \cdot \alpha}{\sqrt{2}}
$$

leading to a contradiction.

This result is essentially tight. For any $\delta>0$ consider the matrix:

$$
\alpha \frac{\sqrt{2}-\delta}{n-1} \cdot\left(\begin{array}{cccccc}
0 & 1 & 2 & \cdots & n-2 & n-1 \\
n-1 & 0 & 1 & \cdots & n-3 & n-2 \\
\vdots & \vdots & \vdots & \vdots & \vdots & \vdots \\
1 & 2 & 3 & \cdots & n-1 & 0
\end{array}\right),
$$

where every column sum is $<(n-1) \alpha / \sqrt{2}$ and for every row $i$ and nonempty $I \subseteq[n] \backslash\{i\}$ :

$$
\frac{|I|}{\max _{j \in I} a_{i, j}} \leq \frac{|I|}{a_{i, i_{|I|+1}}}=\frac{|I|}{\alpha(\sqrt{2}-\delta) \frac{|I|}{n-1}}=\frac{n-1}{\alpha(\sqrt{2}-\delta)} .
$$




\section{B Proof of Theorem 9}

Without loss of generality, we assume that mechanism $\mathcal{M}$ allocates each task independently by running the same single-task mechanism for every task. The reason the analysis carries over is that we will only use tasks drawn from a finite pool. Restricted to these profiles of true processing times, there are only finitely many essentially different single-task mechanisms, when the difference is measured from the perspective of allocations. Therefore, even if the taskindependent mechanism $\mathcal{M}$ used a different mechanism for every task, we could always find $n$ single-component mechanisms operating the same way. For a rigorous treatment, we refer the reader to Lemma 6.

We aim to identify some "weakness" of $\mathcal{M}$ by discovering, for canonical cost vectors that are permutations of the true processing times $(1, x, \infty, \infty, \ldots)$, just how much larger $x$ can get, so that there exists some equilibrium under which the task is allocated to the slow machine. We refer to the machine with processing time 1 as the fast machine and to the other, which does not have a processing time of $\infty$, as the slow machine. As the mechanism is not anonymous, there might be a different $x$ for every permutation.

Formally, fix $\frac{\alpha}{(n-1) \sqrt{2}} \geq \varepsilon>0$ and for $i, j=1, \ldots, n$ and $i \neq j$, let

$$
a_{i, j}=\max _{k \in \mathbb{N}}\left\{k \cdot \varepsilon \mid \text { for } \mathbf{t}=(\ldots, \underbrace{1}_{i}, \ldots, \underbrace{k \cdot \varepsilon}_{j}, \ldots), \exists \mathbf{s}_{i, j} \text { s.t. machine } j \text { is allocated the task }\right\}
$$

be the maximum processing time of the slow machine, when the fast machine has index $i$, the slow machine has index $j$ and the slow machine can still receive the task for some equilibrium. To ensure that Lemma 6 can be applied, we need to discretize the processing times, in order to have a finite number of possible tasks. Let $\bar{a}_{i, j}=a_{i, j}+\varepsilon$. Clearly, if $a_{i, j}$ is replaced by $\bar{a}_{i, j}$ in its canonical cost vector then the only allocation remaining will be to give the task to the fast machine. For convenience, we also set $a_{i, i}=\bar{a}_{i, i}=0$. Let $\mathbf{h}_{i, j}$ be the permutation of the canonical cost vector where $i$ is the fast machine and $j$ the slow machine with cost $a_{i, j}$ and $\overline{\mathbf{h}}_{i, j}$ the same but with cost $\bar{a}_{i, j}$ instead.

Next, observe that $0<a_{i, j}<(n-1) \alpha / \sqrt{2}+1$ for all $i \neq j$. The upper bound is due to the assumption on the PoA, which would otherwise be violated for $\mathbf{h}_{i, j}$. For the lower bound, the instance $\overline{\mathbf{h}}_{i, j}$ would have $\mathrm{PoA} \geq 1 / \varepsilon$, leading to a contradiction, for some small enough $\varepsilon$.

For every machine $j$, we create the following profile of true processing times:

$$
\mathbf{t}(j)=\left(\mathbf{h}_{1, j}, \ldots, \mathbf{h}_{(i-1), j}, \mathbf{f}^{\star}(j), \mathbf{h}_{(i+1), j}, \ldots, \mathbf{h}_{n, j}\right)^{\top},
$$

where $\mathbf{f}^{\star}$ contains only one entry that is not $\infty$, at row $j$. Presented in a more convenient matrix form:

$$
\left.\left(\begin{array}{ccccccccc}
1 & \infty & \infty & \infty & \infty & \cdots & \infty & \infty & \infty \\
\infty & 1 & \infty & \infty & \infty & \cdots & \infty & \infty & \infty \\
\vdots & \ddots & \ddots & \ddots & \ddots & \ddots & \ddots & \ddots & \infty \\
\infty & \infty & \cdots & 1 & \infty & \infty & \cdots & \infty & \infty \\
a_{1, j} & a_{2, j} & \cdots & a_{(j-1), j} & 1 & a_{(j+1), j} & \cdots & a_{(n-1), j} & a_{n, j} \\
\infty & \infty & \cdots & \infty & \infty & 1 & \infty & \cdots & \infty \\
\vdots & \vdots & \vdots & \vdots & \vdots & \ddots & \ddots & \ddots & \vdots \\
\infty & \infty & \cdots & \infty & \infty & \infty & \infty & 1 & \infty \\
\infty & \infty & \cdots & \infty & \infty & \infty & \infty & \infty & 1
\end{array}\right)\right\} n,
$$

where machine $j$ is the slow machine for all the tasks and for each task there exists exactly one, distinct, fast machine. By task-independence, the profile of reported processing times

$$
\left(\mathbf{s}_{1, j}, \mathbf{s}_{2, j}, \ldots, \mathbf{s}_{(i-1), j}, \mathbf{f}_{E Q}^{\star}, \mathbf{s}_{(i+1), j}, \ldots, \mathbf{s}_{n, j}\right)^{\top}
$$


is an equilibrium in which all tasks are allocated to the slow machine and $\mathbf{f}_{E Q}^{\star}$ is any equilibrium for task $j$, which clearly has to select the $j$-th machine. Since the optimal makespan is 1 , by the given bound on the PoA, we have that for all $j$ :

$$
\sum_{i=1}^{n} a_{i, j}+1<\frac{(n-1) \cdot \alpha}{\sqrt{2}}+1 \Longrightarrow \sum_{i=1}^{n} a_{i, j}<\frac{(n-1) \cdot \alpha}{\sqrt{2}}
$$

In the proof of Theorem 6, in order to obtain the bound for the PoS, we swapped the positions of the slow and fast machines on each column, which was facilitated by the assumption that the mechanism in that case was anonymous. In this case however mechanism $\mathcal{M}$ is not anonymous, so we have to make a slight adjustment and change $a_{i, j}$ to $\bar{a}_{i, j}$. To obtain a lower bound on the PoS, we instead fix $i$ and using the true processing times

$$
\overline{\mathbf{t}}(i)=\left(\overline{\mathbf{h}}_{i, 1}, \ldots, \overline{\mathbf{h}}_{i,(i-1)}, \overline{\mathbf{f}}^{\star}, \overline{\mathbf{h}}_{i,(i+1)}, \ldots, \overline{\mathbf{h}}_{i, n}\right)^{\top},
$$

we get a matrix similar to the previous one, i.e.,

$$
\left(\begin{array}{ccccccccc}
\bar{a}_{i, 1} & \infty & \infty & \infty & \infty & \cdots & \infty & \infty & \infty \\
\infty & \bar{a}_{i, 2} & \infty & \infty & \infty & \cdots & \infty & \infty & \infty \\
\vdots & \ddots & \ddots & \ddots & \ddots & \ddots & \ddots & \ddots & \infty \\
\infty & \infty & \cdots & \bar{a}_{i,(i-1)} & \infty & \infty & \cdots & \infty & \infty \\
1 & 1 & \cdots & 1 & \max _{j} \bar{a}_{i, j} & 1 & \cdots & 1 & 1 \\
\infty & \infty & \cdots & \infty & \infty & \bar{a}_{i,(i+1)} & \infty & \cdots & \infty \\
\vdots & \vdots & \vdots & \vdots & \vdots & \ddots & \ddots & \ddots & \vdots \\
\infty & \infty & \cdots & \infty & \infty & \infty & \infty & \bar{a}_{i,(n-1)} & \infty \\
\infty & \infty & \cdots & \infty & \infty & \infty & \infty & \infty & \bar{a}_{i, n}
\end{array}\right)
$$

Again, by task independence and the definition of $\bar{a}_{i, j}$, the mechanism has to allocate each task to the fast machine, which for all tasks is machine $i$. The optimal allocation could potentially only allocate to slow machines and produce an allocation with makespan at $\operatorname{most}_{\max } \bar{a}_{i, j}$. The lower bound on the PoS can be further improved by restricting the input to only contain a nonempty subset $I \subseteq[n] \backslash\{i\}$ of the tasks, plus task $i$ which is special. This reduces the makespan of both $\mathcal{M}$ and the optimal, but the overall fraction could increase. Notice that task $i$ (which has only one entry which is not $\infty$, adjusted to be the maximum amongst the $|I|$ selected $\left.\bar{a}_{i, j}\right)$ is always added, as it increases $\mathcal{M}$ 's makespan for free. In particular:

$$
\operatorname{PoS} \geq \max _{I \subseteq[n] \backslash\{i\}} \frac{|I|+\max _{j \in I} \bar{a}_{i, j}}{\max _{j \in I} \bar{a}_{i, j}}=\max _{I \subseteq[n] \backslash\{i\}} \frac{|I|}{\max _{j \in I} \bar{a}_{i, j}}+1
$$

Notice that the $a_{i, j}$ satisfy the premises of Lemma 5: the 'if' part holds because each $\mathbf{t}(j)$ generates one such inequality by the PoA bound. Similarly, any inequality generated by the 'then' part can be captured by an appropriate PoS bound in $\overline{\mathbf{t}}(i)$. Applying the result of Lemma 5 to Eq. (3) we get:

$$
\operatorname{PoS} \geq \max _{I \subseteq[n] \backslash\{i\}} \frac{|I|}{\max _{j \in I} \bar{a}_{i, j}}+1=\max _{I \subseteq[n] \backslash\{i\}} \frac{|I|}{\max _{j \in I} a_{i, j}+\varepsilon}+1>\frac{n-1}{\alpha \sqrt{2}}+1,
$$

which completes the proof.

The proof of Theorem 9 shows that foregoing anonymity cannot lead to mechanisms with asymptotically tighter Pareto frontiers. Moreover, it leads to the following observations. Most likely, if there exists a stronger bound, it cannot be obtained with our canonical cost vectors and would need to use a richer input. At the same time, if a non-anonymous mechanism that achieves a better trade-off than that given by Theorem 6 does exist, the matrix at the end of Lemma 5 could provide some insight on what that mechanism could look like, at least when restricted to these tasks. 
Lemma 6. Theorem 9 holds for all task-independent mechanisms $\mathcal{M}$.

Proof. Continuing from our note in the beginning of Theorem 9, we show that given a taskindependent mechanism $\mathcal{M}$, it is always possible to find $n$ single-task component mechanisms that behave the same way for the very specific inputs needed for the lower bounds. Notice that in the proof of Theorem 9 the tasks we used all come from a finite set of true processing times $\mathcal{C}$. In particular, $\mathcal{C}$ contains all the permutations of $(1, a, \infty, \infty, \ldots, \infty)$, where $a$ is of the form $k \cdot \varepsilon$ and $0<a<(n-1) \cdot \alpha / \sqrt{2}+\varepsilon$. Therefore:

$$
|\mathcal{C}|=n(n-1) \frac{(n-1) \cdot \alpha / \sqrt{2}}{\varepsilon} .
$$

Fix a single-task mechanism $\mathcal{A}$. For a true processing time vector $\mathbf{t} \in \mathcal{C}$, call machine $j$ accessible from $\mathbf{t}$ if there exists an equilibrium for which the task is assigned to that machine. Let $A C C(\mathbf{t})$ be the set of accessible machines for component $\mathcal{A}$ for that $\mathbf{t}$ and $\mathcal{B}(\mathcal{A})$ be the behaviour set of $\mathcal{A}$, defined as

$$
\mathcal{B}(\mathcal{A})=\{(\mathbf{t}, A C C(\mathbf{t})) \mid \mathbf{t} \in \mathcal{C}\}
$$

This fully characterizes $\mathcal{A}$ from the perspective of accessible allocations for tasks in $\mathcal{C}$. Note that for two different single-task mechanisms $\mathcal{A}_{1}$ and $\mathcal{A}_{2}$, it could be that $\mathcal{B}\left(\mathcal{A}_{1}\right)=\mathcal{B}\left(\mathcal{A}_{2}\right)$ without them being the same mechanism: they must however reach the same allocations given the same $\mathbf{t}$ (potentially through different equilibria).

Clearly, $A C C(\mathbf{t})$ can be one of $2^{n}-1$ possible sets. This is because $A C C(\mathbf{t})$ can be any subset of the $n$ machines, except the empty subset, since each task has to be allocated to some machine at every equilibrium. This is however all we need in Theorem 9. Therefore, the total number of behaviour sets is at most:

$$
\left(2^{n}-1\right)^{|\mathcal{C}|}
$$

which is quite large, but bounded.

Let $\mathcal{A}_{j}$ be the single-task mechanism used by mechanism $\mathcal{M}$ for the $j$ 'th task, given an input with $n\left(2^{n}-1\right)^{|\mathcal{C}|}$ tasks from $\mathcal{C}$ in total. By the pidgeonhole principle, there must be at least $n$ such single-task mechanisms with the same behaviour set. Setting all other tasks to have real processing times 0 for all tasks, we have successfully extracted a mechanism $\mathcal{M}^{\prime}$ that behaves exactly as needed for Theorem 9 . 